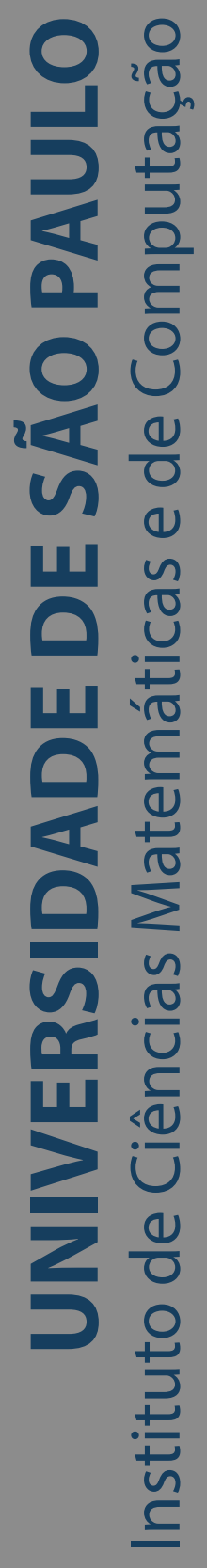

\title{
Derivações simples de álgebras afins
}

\section{Leonardo Soares Moço}

Dissertação de Mestrado do Programa de Pós-Graduação em Matemática (PPG-Mat) 

Assinatura:

\title{
Leonardo Soares Moço
}

\section{Derivações simples de álgebras afins}

\begin{abstract}
Dissertação apresentada ao Instituto de Ciências Matemáticas e de Computação - ICMC-USP, como parte dos requisitos para obtenção do título de Mestre em Ciências - Matemática. VERSÃO REVISADA
\end{abstract}

Área de Concentração: Matemática

Orientador: Prof. Dr. Daniel Levcovitz 
Ficha catalográfica elaborada pela Biblioteca Prof. Achille Bassi e Seção Técnica de Informática, ICMC/USP, com os dados inseridos pelo(a) autor(a)

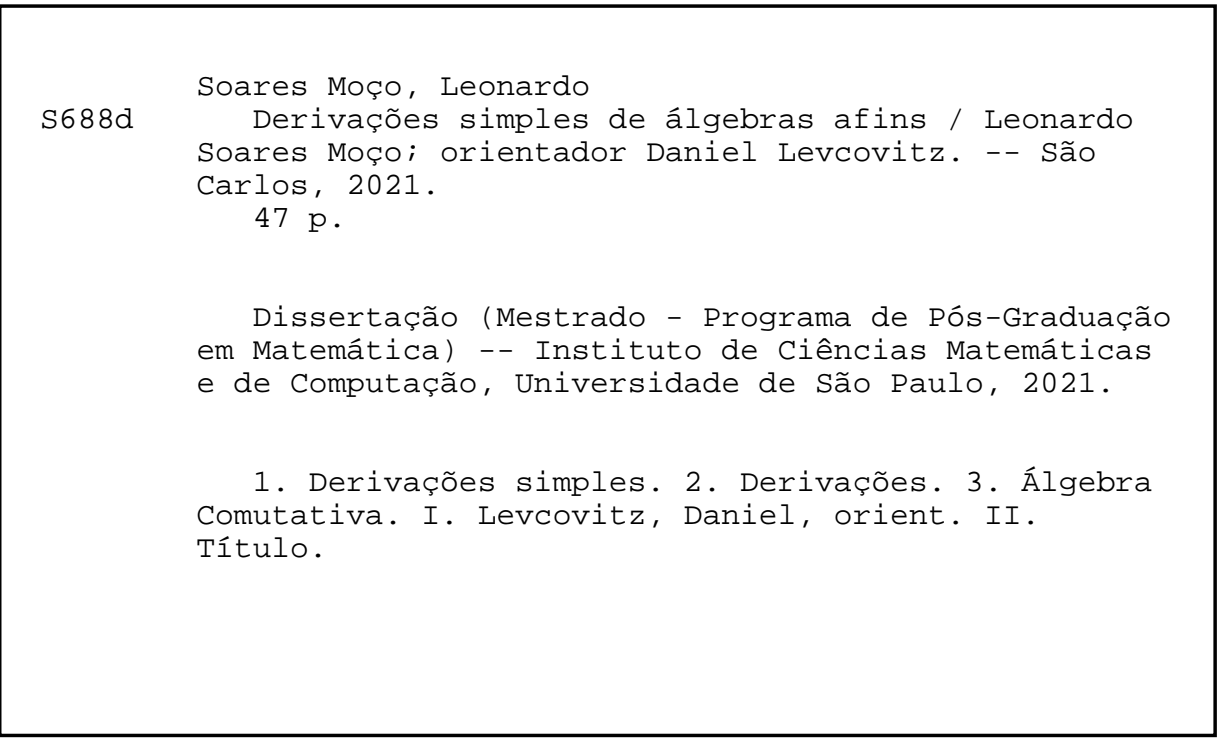

Bibliotecários responsáveis pela estrutura de catalogação da publicação de acordo com a AACR2: Gláucia Maria Saia Cristianini - CRB - 8/4938 Juliana de Souza Moraes - CRB - 8/6176 


\section{Leonardo Soares Moço}

\section{Simple derivations of affine algebras}

Dissertation submitted to the Instituto de Ciências Matemáticas e de Computação - ICMC-USP - in accordance with the requirements of the Mathematics Graduate Program, for the degree of Master in Science. FINAL VERSION

Concentration Area: Mathematics

Advisor: Prof. Dr. Daniel Levcovitz 

Dedico esse trabalho à minha família e à Universidade Pública Brasileira 

Ao longo dessa jornada pude contar com várias pessoas especiais as quais sou imensamente grato. Meus pais, Luciana e Leonel, além de toda a minha família, que sempre me apoiaram. Os amigos, sejam eles da graduação, do Kendo ou dos jogos que me ajudaram tanto nos momentos de estudo antes das provas, quanto nos momentos de descanso que tornaram essa jornada mais leve. E especialmente o meu orientador, Daniel Levcovitz, que tem me acompanhado desde o inicio da graduação, me mostrando os encantos que a álgebra esconde.

Também sou imensamente grato às instituições que permitiram que eu traçasse o caminho acadêmico, em especial o Instituto de Matemática Pura e Aplicada (IMPA) que graças a iniciativas como a Olimpíada Brasileira de Matemática das Escolas Pública (OBMEP) e o Programa de Iniciação Científica júnior (PICjr), tem divulgado e tornado acessível a matemática pura a alunos do ensino básico público brasileiro, que sem isso não teriam tal oportunidade e a Universidade de São Paulo (USP) por toda a estrutura física e de pessoal que permitem o desenvolvimento da ciência nacional.

O presente trabalho foi realizado com apoio da Coordenação de Aperfeiçoamento de Pessoal de Nível Superior - Brasil (CAPES) - Código de Financiamento 001. O qual sou imensamente agradecido. 

"São as perguntas que não sabemos responder que mais nos ensinam. Elas nos ensinam a pensar. Se você dá uma resposta a um homem, tudo o que ele ganha é um fato qualquer. Mas, se você lhe der uma pergunta, ele procurará suas próprias respostas." 



\section{RESUMO}

MOCO, L. S. Derivações simples de álgebras afins. 2021. 47 p. Dissertação (Mestrado em Ciências - Matemática) - Instituto de Ciências Matemáticas e de Computação, Universidade de São Paulo, São Carlos - SP, 2021.

As derivações que não deixam ideais invariantes no anel de polinômios em uma variável sobre um corpo $K$ são bem conhecidas, entretanto, quando estamos sobre duas ou mais variáveis ou sobre quocientes dessas álgebras polinomiais ainda não é possível caracterizá-las. Dada uma derivação $d$ sobre uma $K$-álgebra $R$, são apresentados nesse trabalho alguns resultados a respeito da $d$-simplicidade de $R$, isto é, da ausência de ideais de $R$ não triviais que são invariantes por $d$. Entre eles citamos o teorema de Shamsuddin e a correspondência entre $d$-simplicidade e o comportamento de espaços tangentes de conjuntos algébricos afins. A parte mais importante, o capítulo 4, apresenta alguns exemplos geométricos que ilustram bem propriedades de subconjuntos algébricos afins relacionadas à derivações de seu anel de coordenadas. Os exemplos e resultados aqui apresentados fazem parte da tese de doutorado de J. Archer (ARCHER, 1981).

Palavras-chave: Álgebra comutativa, Derivações, Derivações simples. 



\section{ABSTRACT}

MOCO, L. S. Simple derivations of affine algebras. 2021. 47 p. Dissertação (Mestrado em Ciências - Matemática) - Instituto de Ciências Matemáticas e de Computação, Universidade de São Paulo, São Carlos - SP, 2021.

The derivations that do not leave invariant ideals are well known for the case of polynomial rings in one variable over a field $K$. However, over two or more variables or over quotients of polynomial algebras, it is still not possible to classify them. Given a derivation $d$ on a $K$ algebra $R$, this work presents some results regarding the $d$-simplicity of $R$, that is, the absence of non-trivial $R$ ideals that are invariant by the action of $d$, like Shamsuddin's theorem, and correspondences between such property and the behavior of tangent spaces to affine algebraic sets. The most important part, chapter 4, has some geometric examples that well illustrate properties of affine algebraic subsets related to derivations of their coordinate ring. The examples and results presented here are part of J. Archer's doctoral thesis (ARCHER, 1981).

Keywords: Commutative algebra, Derivations, Simple derivations. 



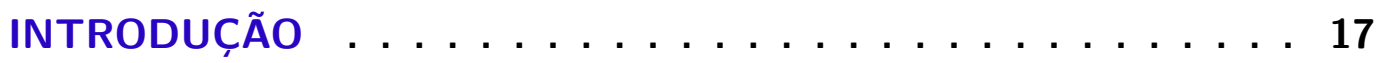

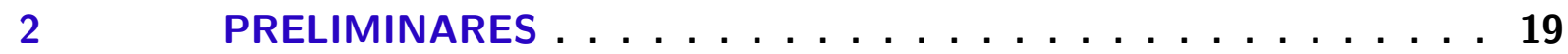

$2.0 .1 \quad$ Derivações . . . . . . . . . . . . . . . . . . . . 19

2.0.2 Estendendo derivações em anéis locais . . . . . . . . . . . . . 23

2.0.3 Derivações em corpos . . . . . . . . . . . . . . . . . . . . 24

2.0.4 Geometria Algébrica . . . . . . . . . . . . . . . 24

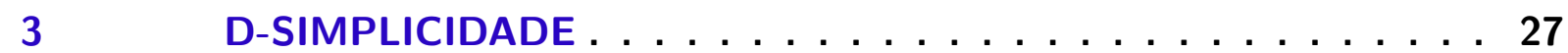

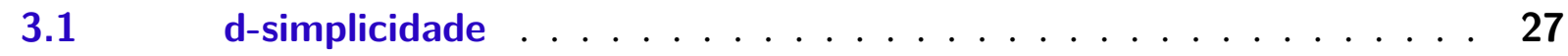

$3.2 \quad$ Lema de Zariski . . . . . . . . . . . . . . . . . . . 28

$3.3 \mathrm{~d}$-simplicidade em extensões inteiras . . . . . . . . . 31

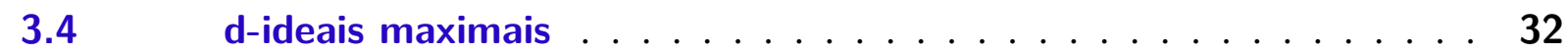

$3.5 \quad$ Adjuntando uma variável . . . . . . . . . . . . . 33

4 D-SIMPLICIDADE DE ÁlgEBRAS AFINS . . . . . . . . . . . . 37

$4.1 \quad$ d-simplicidade em anéis de polinômios $\ldots \ldots \ldots \ldots$

$4.2 \quad$ Exemplos geométricos . . . . . . . . . . . . . . 38

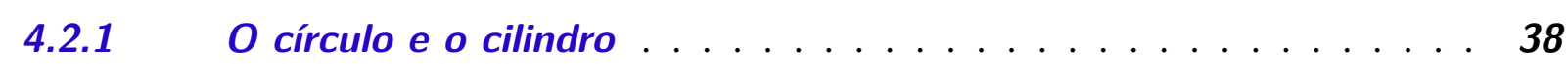

$4.2 .2 \quad 0$ toro real . . . . . . . . . . . . . . . . . . 40

$4.2 .3 \quad$ Hipersuperfícies ................... 43

REFERÊNCIAS . . . . . . . . . . . . . . . . . . . 47 



\section{1}

\section{INTRODUÇÃO}

Seja $R$ um anel comutativo e $d$ uma derivação de $R$. Dizemos que $d$ é uma derivação simples de $R$ (ou apenas que $R$ é $d$-simples) se $R$ não tiver nenhum ideal $I$, não trivial, tal que $d(I) \subseteq I$. Tal ideal $I$ é chamado de ideal $d$-invariante, ideal $d$-estável ou simplesmente um $d$-ideal.

Quando o anel $R$ for uma $K$-álgebra, para algum corpo $K$ de caraterística zero, dizemos que $d$ é uma $K$-derivação de $R$ se $d$ se anula sobre $K$, isto é, $d(K)=0$.

A pesquisa sobre derivações simples de $K$-álgebras comutativas aumentou muito nos últimos anos. Isso se deve, em parte, às diversas conexões que derivações simples têm com outras áreas da matemática. Vamos citar algumas delas. Derivações simples aparecem na teoria dos anéis não comutativos noetherianos simples. De fato, se $R[X ; d]$ é uma extensão de Ore de $R$ por $d$, então $R[X ; d]$ é um anel simples (no sentido de que não tem ideais bilaterais não triviais) se, e somente se, $R$ for $d$-simples (ver (GOODEARL; WARFIELD, 2004)). Neste caso, se $R$ é uma $\mathrm{K}$-álgebra noetheriana, então $R[X ; d]$ também o é (pelo teorema da base de Hilbert) e $R[X ; d]$ fornece um exemplo útil para testar conjecturas na teoria dos anéis simples noetherianos não comutativos.

Por outro lado, são muitas as aplicações de $d$-simplicidade em álgebra comutativa e geometria algébrica. Entre elas destacam-se aplicações na teoria de folheações holomorfas (ver, por exemplo, (COUTINHO, 2008), (COUTINHO; LEVCOVITZ, 2014) e (OLIVEIRA, 2012)), $\mathscr{D}$-módulos (ver, por exemplo, (COUTINHO, 2013) e (COUTINHO, 2007) ) e até mesmo na questão da independência algébrica de soluções de certas equações diferenciais no anel da série de potências formais $K[[t]]$ (ver (BRUMATTI; LEQUAIN; LEVCOVITZ, 2003)).

Apesar de sua ubiquidade, derivações simples estão longe de serem bem compreendidas e até mesmo uma caracterização das derivações simples do anel de polinômios $K\left[x_{1}, \cdots, x_{n}\right]$ não é conhecida até agora (o único caso conhecido, que é trivial, é quando $n=1$ ).

Enquanto que no caso do anel de polinômios $K\left[x_{1}, \cdots, x_{n}\right]$ (sempre sobre um corpo $K$ de 
característica zero) já temos, hoje em dia, diversos exemplos e mesmo famílias de derivações simples; para álgebras afins não polinomiais (isto é, quocientes próprios de anéis de polinômios) pouco são os exemplos de derivações simples conhecidos. Um dos objetivos desta dissertação é justamente exibir alguns destes exemplos, todos extraídos da tese de doutorado de Archer (ARCHER, 1981). Na verdade, além destes exemplos "geométricos", selecionamos outros resultados desta referência principal para por na dissertação.

Assim, o capítulo 2 trata dos preliminares que serão utilizados nos próximos capítulos. Aqui expomos, quase sem nenhuma prova, alguns fatos básicos sobre derivações e suas extensões. Fazemos também um breve resumo de resultados básicos de geometria algébrica que serão usados depois.

No capítulo 3 provamos o importante Lema de Zariski sobre derivações em anéis completos. Também provamos vários resultados sobre derivações simples em anéis de polinômios. Em particular, mostramos o importante teorema de Shamsuddin que é, até hoje, a maior fonte de exemplos de derivações simples em anéis de polinômios. Neste capítulo explorarmos também a conexão entre a $d$-simplicidade de $R$ e o fato do módulo de diferenciais de Kähler de $R, \Omega_{K}(R)$, possuir um somando direto livre de posto um.

Finalmente no capítulo 4, o mais importante, exibimos vários exemplos "geométricos"de derivações simples em álgebras afins.

Importante salientar aqui que todo o material desta dissertação faz parte da belíssima tese de doutorado de Archer (ARCHER, 1981) cuja leitura foi precisamente o projeto deste mestrado. 


\subsubsection{Derivações}

Definição 1. Sejam $R$ um anel comutativo e $M$ um $R$-módulo. Um homomorfismo $d: R \rightarrow M$ de grupos aditivos é uma derivação de $R$ em $M$ se satisfaz a regra de Leibniz, isto é:

$$
d\left(r_{1} r_{2}\right)=r_{1} d\left(r_{2}\right)+d\left(r_{1}\right) r_{2} \text { para todo } r_{1}, r_{2} \in R \text {. }
$$

Suponha que $R$ é uma $k$-álgebra, onde $k$ é um anel comutativo, com homomorfismo estrutural $\theta: k \rightarrow R$. Dizemos que uma derivação $d: R \rightarrow M$ é uma $k$-derivação se $d \circ \theta$ se anula em $k$.

Se $T$ é um anel comutativo, um homomorfismo de anéis $\phi: R \rightarrow T$ induz uma estrutura de $R$-módulo em $T$. As derivações de $R$ em $T$ com essa estrutura de $R$-módulo são chamadas de derivações de tipo $\phi$ e satisfazem

$$
d\left(r_{1} r_{2}\right)=\phi\left(r_{1}\right) d\left(r_{2}\right)+d\left(r_{1}\right) \phi\left(r_{2}\right) \text { para todo } r_{1}, r_{2} \in R
$$

Denotamos o conjunto de todas as derivações de $R$ em $M$ por $\operatorname{Der}(R, M)$, o conjunto de todas as $k$-derivações de $R$ em $M$ por $\operatorname{Der}_{k}(R, M)$ e o conjunto das derivações de tipo $\phi$ de $R$ em $T$ por $\operatorname{Der}(R, T, \phi)$. Quando $R=M$ escrevemos simplesmente $\operatorname{Der}(R)$ e $\operatorname{Der}_{k}(R)$. De forma similar denotamos o conjunto das $k$-derivações de tipo $\phi$ de $R$ em $T$ por $\operatorname{Der}_{k}(R, T, \phi)$.

Observação 2. Os conjuntos $\operatorname{Der}(R, M)$ e $\operatorname{Der}_{k}(R, M)$ são $R$-módulos. Os conjuntos $\operatorname{Der}(R, T, \phi)$ e $\operatorname{Der}_{k}(R, T, \phi)$ são $R$-módulos e $T$-módulos.

Exemplo 3. Seja $k$ um corpo. No anel de polinômios em $n$ variáveis, $R=k\left[X_{1}, \cdots, X_{n}\right]$ a derivada parcial usual do cálculo em relação a variável $X_{i}$, denotada por $\frac{\partial}{\partial X_{i}}$ para $i=1, \cdots, n$ é uma derivação de $R$ em $R$. 
Proposição 4. Sejam $k$ um anel e $R, S$ e $T$-álgebras. Sejam $\theta: R \rightarrow S$ e $\phi: S \rightarrow T$ e considere

$$
\begin{gathered}
F=\left\{d \in \operatorname{Der}_{k}(R) ; d(\operatorname{Ker}(\theta)) \subseteq \operatorname{Ker}(\theta)\right\}, \\
G=\left\{d \in \operatorname{Der}_{k}(R, S, \theta) ; d(\operatorname{Ker}(\theta))=0\right\} \mathrm{e} \\
H=\left\{d \in \operatorname{Der}_{k}(R, T, \phi \circ \theta) ; d(\operatorname{Ker}(\theta))=0\right\} .
\end{gathered}
$$

Então

1. O morfismo $\operatorname{Der}_{k}(R) \rightarrow \operatorname{Der}_{k}(R, S, \theta)$ definido por $d \mapsto \theta \circ d$ é um homomorfismo de $R$-módulos de $F$ em $G$

2. Se $\theta$ for sobrejetor, então existe um homomorfismo de $k$-módulos de $F$ em $\operatorname{Der}_{k}(S)$.

3. O morfismo $h: \operatorname{Der}_{k}(S, T, \phi) \rightarrow \operatorname{Der}_{k}(R, T, \phi \circ \theta)$ definido por $h(D)=D \circ \theta$ é um homomorfismo de $T$-módulos e leva $\operatorname{Der}_{k}(S, T, \phi)$ em $H$. Além disso, se $\theta$ for sobrejetor então $h$ é um isomorfismo de $T$-módulos de $\operatorname{Der}_{k}(S, T, \phi)$ em $\mathrm{H}$.

Demonstração: 1) É simples verificar que $F$ e $G$ são $R$-módulos e que o morfismo $\operatorname{Der}_{k}(R) \rightarrow \operatorname{Der}_{k}(R, S, \theta)$ dado por $d \mapsto \theta \circ d$ é um homomorfismo de $R$-módulos pois $\theta \circ(r d)(x)=\theta(r) \theta(d(x))$. Tome agora $d \in F$, então $d(\operatorname{Ker}(\theta)) \subseteq \operatorname{Ker}(\theta)$ e consequentemente $\theta \circ d(\operatorname{Ker}(\theta)) \subseteq \theta(\operatorname{Ker}(\theta))=0, \log 0 \theta \circ d \in G$.

2) Se $\theta$ é sobrejetor, então todo elemento de $S$ pode ser escrito como $\theta(r)$ para algum $r \in R$. Seja $d \in F$, defina um morfismo $\bar{d}: S \rightarrow S$ por $\bar{d}(\theta(r))=\theta \circ d(r)$. Veja que $\bar{d}$ está bem definido, pois se $\theta\left(r_{1}\right)=\theta\left(r_{2}\right)$, então $\theta\left(r_{1}-r_{2}\right)=0 \Rightarrow r_{1}-r_{2} \in \operatorname{Ker}(\theta), \operatorname{logo} \bar{d}\left(\theta\left(r_{1}-\right.\right.$ $\left.\left.r_{2}\right)\right)=\theta \circ d\left(r_{1}-r_{2}\right)$, como $d \in F$ então $d\left(r_{1}-r_{2}\right) \in \operatorname{Ker}(\theta)$, portanto $\bar{d}\left(\theta\left(r_{1}-r_{2}\right)\right)=0$ e $\bar{d}\left(\theta\left(r_{1}\right)\right)=\bar{d}\left(\theta\left(r_{2}\right)\right.$. Além disso $\bar{d} \in \operatorname{Der}_{k}(S)$, pois

$$
\bar{d}\left(\theta\left(r_{1}\right) \theta\left(r_{2}\right)\right)=\theta \circ d\left(r_{1} r_{2}\right)=\theta \circ d\left(r_{1}\right) \theta\left(r_{2}\right)+\theta\left(r_{1}\right) \theta \circ d\left(r_{2}\right)=\bar{d}\left(r_{1}\right) \theta\left(r_{2}\right)+\theta\left(r_{1}\right) \bar{d}\left(r_{2}\right) .
$$

Por fim, a aplicação $f: F \rightarrow \operatorname{Der}_{k}(S)$ definida por $f(d)=\bar{d}$ é um homomorfismo de $k$-módulos.

3) A aplicação $h: \operatorname{Der}_{k}(S, T, \phi) \rightarrow \operatorname{Der}_{k}(R, T, \phi \circ \theta)$ é claramente um homomorfismo de $T$-módulos. Além disso $D \circ \theta(\operatorname{Ker}(\theta))=0$, para todo $D \in \operatorname{Der}_{k}(S, T, \phi)$, portanto a imagem de $h$ está contida em $\mathrm{H}$.

Suponha agora que $\theta$ é sobrejetor, então todo elemento de $S$ é da forma $\theta(r)$ para algum $r \in R$. Dada $d \in H$, defina a aplicação $D: S \rightarrow T$ por $D(\theta(r))=d(r)$. Note que $D$ está bem definida pois $d(\operatorname{Ker}(\theta))=0$. Além disso é fácil verificar, como no item anterior, que $D \in D e r_{k}(S, T, \phi)$ e $h(D)=d$, portanto $h$ é uma sobrejeção de $\operatorname{Der}_{k}(S, T, \phi)$ em $H$. Suponha agora que $D \in \operatorname{Ker}(h)$. Então $D \circ \theta=0$ e como $S=\theta(R)$ temos que $D(S)=D(\theta(R))=D \circ \theta(R)=0$, portando $D=0$ e $\operatorname{Ker}(h)=0$. Concluímos com isso que $h$ é um isomorfismo de $T$-módulos. 
Observação 5. Mantendo a notação usada na proposição anterior, com $\theta: R \rightarrow S$ sobrejetor e $f: F \rightarrow \operatorname{Der}_{k}(S)$ dada por $d \mapsto \bar{d}$. Suponha que $I \subseteq S$ e $J \subseteq R$ são ideais, tais que $\operatorname{Ker}(\theta) \subseteq J$ e $\theta(J)=I$. Então $\bar{d}(I) \subseteq I$ se, e somente se, $d(J) \subseteq J$. Portanto os ideais de $S$ que são invariantes por $\bar{d}$ estão em correspondência biunívoca com os ideais de $R$, contendo $\operatorname{Ker}(\theta)$ que são invariantes por $d$.

Proposição 6. Sejam $k$ um anel, $X_{1}, \ldots, X_{n}$ variáveis e $R=k\left[X_{1}, \ldots, X_{n}\right]$. Seja $S$ uma $k$-álgebra e $\theta: R \rightarrow S$ um homomorfismo de $k$-álgebras. Então $\operatorname{Der}_{k}(R, S, \theta)$ é um $S$-módulo livre com base $\left\{\theta \circ \frac{\partial}{\partial X_{1}}, \ldots, \theta \circ \frac{\partial}{\partial X_{n}}\right\}$.

Demonstração: $\operatorname{Seja} d \in \operatorname{Der}_{k}(R, S, \theta)$. Quando $n=1$ é fácil ver que $d\left(X_{1}^{a}\right)=$ $d\left(X_{1}\right) \theta \circ \frac{\partial X_{1}^{a}}{\partial X_{1}}$ (por indução em $a$, usando a regra de Leibniz). Vamos agora supor que para $n-1$ variáveis vale que, para todo monômio $g=X_{1}^{a_{1}} \cdots \cdot X_{n-1}^{a_{n-1}}$ temos que

$$
d(g)=\sum_{i=1}^{n-1} d\left(X_{i}\right) \theta \circ \frac{\partial g}{\partial X_{i}}
$$

Adicionando uma nova variável $X_{n}$ com grau $a_{n}$ ao monômio obtemos que

$$
\begin{gathered}
d\left(g . X_{n}^{a_{n}}\right)=d(g) \theta\left(X_{n}^{a_{n}}\right)+\theta(g) d\left(X_{n}^{a_{n}}\right)=\sum_{i=1}^{n-1} d\left(X_{i}\right) \theta\left(X_{n}^{a_{n}}\right) \theta \circ \frac{\partial g}{\partial X_{i}}+\theta(g) d\left(X_{n}\right) \theta \circ \frac{\partial X_{n}^{a_{n}}}{\partial X_{n}} \\
=\sum_{i=1}^{n-1} d\left(X_{i}\right) \theta\left(\frac{\partial g X_{n}^{a_{n}}}{\partial X_{i}}\right)+d\left(X_{n}\right) \theta \frac{\partial g X_{n}^{a_{n}}}{\partial X_{n}}=\sum_{i=1}^{n} d\left(X_{i}\right) \theta \circ \frac{\partial g X_{n}^{a_{n}}}{\partial X_{i}} .
\end{gathered}
$$

Portanto, usando a linearidade de $d$ obtemos que dado um polinômio qualquer $f \in R$ temos que

$$
d(f)=\sum_{i=1}^{n} d\left(X_{i}\right) \theta \circ \frac{\partial f}{\partial X_{i}} .
$$

Concluímos com isso que $\left\{\theta \circ \frac{\partial}{\partial X_{1}}, \ldots, \theta \circ \frac{\partial}{\partial X_{n}}\right\}$ gera $\operatorname{Der}(R, S, \theta)$ como um $S$-módulo.

Seja agora $D=\sum_{i=1}^{n} s_{i} \theta \circ \frac{\partial}{\partial X_{i}} \in \operatorname{Der}(R, S, \theta)$, com $s_{i} \in S$. Então $s_{i}=d\left(X_{i}\right)$ para $1 \leq i \leq n$. Logo $D=0$ se e somente se $s_{i}=0 \forall i$ e portanto $\theta \circ \frac{\partial}{\partial X_{1}}, \ldots, \theta \circ \frac{\partial}{\partial X_{n}}$ são linearmente independentes sobre $S$ e $\operatorname{Der}(R, S, \theta)$ é um $S$-módulo livre.

Teorema 7. Seja $k$ um anel, $R=k\left[X_{1}, \ldots, X_{n}\right], I$ um ideal de $R$ e $S=\frac{R}{I}$. Suponha que $I$ pode ser gerado por elementos $f_{1}, \ldots, f_{m}$ e seja $\theta: R \rightarrow S$ a projeção natural. Seja $T$ uma $k$-álgebra e suponha que $\phi: S \rightarrow T$ é um homomorfismo de $k$-álgebras. Considere o seguinte sistema de equações nas variáveis $t_{1}, \ldots t_{n}$ :

$$
\sum_{j=1}^{n} t_{j} \phi \circ \theta \circ \frac{\partial f_{i}}{\partial X_{j}}=0 \quad \text { para todo } 1 \leq i \leq m .
$$

Então o $T$-módulo $M$ das soluções desse sistema é isomorfo a $\operatorname{Der}_{k}(S, T, \phi)$. 
Demonstração: Pelo item 3 da proposição 4 precisamos apenas mostrar que $M$ é isomorfo ao $T$-módulo $H=\left\{D \in \operatorname{Der}_{k}(R, T, \phi \circ \theta) ; D(I)=0\right\}$. Pela proposição anterior $\operatorname{Der}_{k}(R, T, \phi \circ$ $\theta)$ é um $T$-módulo livre gerado por $\phi \circ \theta \circ \frac{\partial}{\partial X_{1}}, \ldots, \phi \circ \theta \circ \frac{\partial}{\partial X_{n}}$.

Seja $d \in \operatorname{Der}_{k}(R, T, \phi \circ \theta)$, então existem únicos $t_{1}, \ldots, t_{n}$ tais que $d=\sum_{j=1}^{n} t_{j} \phi \circ \theta \circ \frac{\partial}{\partial X_{j}}$.

Tome $f \in I$, então $f=\sum_{i=1}^{m} g_{i} f_{i}$ para $g_{i} \in R$ e isso implica que

$$
\begin{gathered}
d(f)=d\left(\sum_{i=1}^{m} g_{i} f_{i}\right)=\sum_{i=1}^{m} d\left(g_{i} f_{i}\right)= \\
=\sum_{i=1}^{m} \underbrace{\left(d\left(g_{i}\right) \phi \circ \theta\left(f_{i}\right)\right.}_{=0}+\phi \circ \theta\left(g_{i}\right) d\left(f_{i}\right))=\sum_{i=1}^{m} \phi \circ \theta\left(g_{i}\right) d\left(f_{i}\right),
\end{gathered}
$$

pois $\theta\left(f_{i}\right)=0$. Portanto $d \in H$ se, e somente se, $d(f)=0 \forall f \in I$ se, e somente se, $d\left(f_{i}\right)=0$ para todo $1 \leq i \leq m$ e isso acontece se, e somente se

$$
\sum_{j=1}^{n} t_{j} . \phi \theta \frac{\partial f_{i}}{\partial X_{j}}=0 \text { para todo } 1 \leq i \leq m
$$

Definição 8. Seja $k$ um anel e $R$ uma $k$-álgebra. Vamos denotar por $\Omega_{k}(R)$ o módulo de $k$ diferenciais de $R$, que é o $R$-módulo que satisfaz:

1. Existe uma $k$-derivação $d_{k}^{R}: R \rightarrow \Omega_{k}(R)$, chamada de $k$-derivação universal de $R$.

2. O módulo $\Omega_{k}(R)$ é gerado sobre $R$ pelo conjunto $\left\{d_{k}^{R}(r) ; r \in R\right\}$.

3. Se $M$ é um $R$-módulo e $d \in \operatorname{Der}_{k}(R, M)$, então existe um único $h \in \operatorname{Hom}_{R}\left(\Omega_{k}(R), M\right)$ tal que $d=h \circ d_{k}^{R}$.

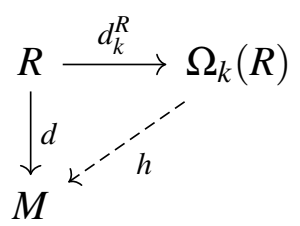

A propriedade 3 é a propriedade universal de $\Omega_{k}(R)$. A existência e unicidade desse módulo é um fato bem conhecido. Uma consequência dessa propriedade universal é o seguinte lema:

Lema 9. Para todo $R$-módulo $M$, temos que $\operatorname{Der}_{k}(R, M) \simeq \operatorname{Hom}_{R}\left(\Omega_{k}(R), M\right)$.

Exemplo 10. Seja $R$ uma $k$-álgebra, $\Lambda$ um conjunto de índices e suponha que $R$ possa ser gerada sobre $k$ pelo conjunto $\left\{x_{i} ; i \in \Lambda\right\}$. Então $d^{R}(r)=\sum_{i \in \Lambda} d^{R}\left(x_{i}\right) \frac{\partial r}{\partial x_{i}}$ para todo $r \in R$ e $\Omega_{k}(R)$ pode ser gerado sobre $R$ por $\left\{d^{R}\left(x_{i}\right) ; i \in \Lambda\right\}$.

Proposição 11. Seja $R=\frac{k\left[X_{1}, \cdots, X_{n}\right]}{\left(f_{1}, \cdots, f_{m}\right)}$ e $\theta: k\left[X_{1}, \cdots X_{n}\right] \rightarrow R$ o homomorfismo natural. Considere o $R$-módulo $T$ gerado por $t_{1}, \cdots, t_{n}$ sujeitos a $m$ relações:

$$
\sum_{j=1}^{n} t_{j} \theta \circ \frac{\partial f_{i}}{\partial X_{j}} \quad \operatorname{com} i=1,2, \cdots, m .
$$


Então $T \cong \Omega_{k}(R)$.

Demonstração: Vamos encontrar uma $k$-derivação universal $d: R \rightarrow T$. Seja $r \in R$, então $r=\theta(f)$ para algum $f \in k\left[X_{1}, \cdots, X_{n}\right]$. Defina uma função $d: R \rightarrow T$ por $\sum_{j=1}^{n} t_{j} \theta \circ \frac{\partial f}{\partial X_{j}}$.

As relações (2.1) garantem que $d$ está bem definida e é fácil ver que $d$ é uma $k$-derivação. Além disso, $d\left(\theta\left(X_{j}\right)\right)=t_{j}$ para $j=1, \cdots, n$ implica que $T$ é gerado como $R$-módulo por $\{d(r) ; r \in R\}$. Seja agora $D: R \rightarrow N$ uma $k$-derivação de $R$ em algum $R$-módulo $N$. Para cada $i=1, \cdots, m$ temos que $D\left(\theta\left(f_{i}\right)\right)=\sum_{j=1}^{n} D\left(\theta\left(X_{j}\right)\right) \theta \circ \frac{\partial f_{i}}{\partial X_{j}}=0$. Defina uma função $h: T \rightarrow N$ por $h\left(\sum_{j=1}^{n} r_{j} t_{j}\right)=\sum_{j=1}^{n} r_{j} D\left(\theta\left(X_{j}\right)\right)$. Note que $h$ está bem definida, pois se $\sum_{j=1}^{n} r_{j} t_{j}=0$ com $r_{j} \in R$ então $\sum_{j=1}^{n} r_{j} t_{j}=\sum_{i=1}^{m} s_{i}\left(\sum_{j=1}^{n} t_{j} \theta \circ \frac{\partial f_{i}}{\partial X_{j}}\right)$ para alguns $s_{i} \in R$ e portanto $h\left(\sum_{j=1}^{n} r_{j} t_{j}\right)=$ $\sum_{i=1}^{m} s_{i}\left(\sum_{j=1}^{n} D\left(\theta\left(X_{j}\right)\right) \theta \circ \frac{\partial f_{i}}{\partial X_{j}}\right)=\sum_{i=1}^{m} s_{i} 0=0$. Claramente $D=h \circ d$. Seja agora $g \in \operatorname{Hom}(T, N)$ tal que $g \circ d=D$, então para cada $j=1, \cdots n$ temos que $g\left(t_{j}\right)=g \circ d\left(\theta\left(X_{j}\right)\right)=D\left(\theta\left(X_{j}\right)\right)=h\left(t_{j}\right)$, portanto $h=g$ e $h$ é único tal que $D=h \circ d$, o que conclui que $\Omega_{k}(R) \cong T$.

\subsubsection{Estendendo derivações em anéis locais}

Sejam $R$ e $S$ anéis e suponha que $U$ e $V$ são subconjuntos multiplicativamente fechados de $R$ e $S$ respectivamente. Considere $\rho: R \rightarrow U^{-1} R$ e $\rho^{\prime}: S \rightarrow V^{-1} S$ os homomorfismos de localização (veja mais detalhes no capítulo 4 do livro (BORGES; TENGAN, 2015)). Os núcleos desses homomorfismos são, respectivamente

$$
I=\{r \in R ; r u=0 \text { para algum } u \in U\} \quad J=\{s \in S ; s v=0 \text { para algum } v \in V\} .
$$

Além disso, se existir um homomorfismo de anéis $\phi: R \rightarrow S$, tal que $\phi(U) \subseteq V$, então podemos estender $\phi$ a um único homomorfismo de anéis $\phi^{\prime}: U^{-1} R \rightarrow V^{-1} S$ definido por $\phi^{\prime}\left(\frac{r}{u}\right)=\frac{\phi(u)}{\phi(r)}$, para todo $r \in R, u \in U$. Temos que $\phi^{\prime}$ está bem definido pois $\phi(I) \subseteq J$. Vamos usar essas notações ao longo de toda a sessão.

Definição 12. Seja $M$ um $U^{-1} R$-módulo. Uma derivação $d^{\prime} \in \operatorname{Der}\left(U^{-1} R, M\right)$ estende a derivação $d \in \operatorname{Der}(R, M)$ se $d^{\prime}\left(\frac{r}{1}\right)=\frac{d(r)}{1}$, para todo $r \in R$.

Uma derivação $d^{\prime} \in \operatorname{Der}\left(U^{-1} R, V^{-1} S, \phi^{\prime}\right)$ estende uma derivação $d \in \operatorname{Der}(R, S, \phi)$ se $d^{\prime}\left(\frac{r}{1}\right)=\frac{d(r)}{1}$ para todo $r \in R$.

Proposição 13. Todo elemento de $\operatorname{Der}(R, M)$ e $\operatorname{Der}(R, S, \phi)$ se estende a um único elemento de $\operatorname{Der}\left(U^{-1} R, M\right)$ e $\operatorname{Der}\left(U^{-1} R, V^{-1} S, \phi^{\prime}\right)$ respectivamente.

Demonstração: Seja $D \in \operatorname{Der}(R, M)$ e $d \in \operatorname{Der}(R, S, \phi)$. Defina aplicações $D^{\prime}: U^{-1} R \rightarrow$ $M$ e $d^{\prime}: U^{-1} R \rightarrow V^{-1} S$ por

$$
D^{\prime}\left(\frac{r}{u}\right)=\frac{D(r)}{u}-\frac{r D(u)}{u^{2}} \text { e } d^{\prime}\left(\frac{r}{u}\right)=\frac{d(r)}{\phi(u)}-\frac{r d(u)}{\phi(u)^{2}} .
$$


Note que $D^{\prime}$ está bem definida, pois $D(I)=0$, para toda $D \in \operatorname{Der}(R, M)$ e $d^{\prime}$ está bem definida pois $d(I) \subseteq J$ para toda $d \in \operatorname{Der}(R, S, \phi)$. Além disso $D^{\prime}$ é uma derivação, pois é um morfismo aditivo e

$$
\begin{gathered}
D^{\prime}\left(\frac{r_{1}}{u_{1}} \frac{r_{2}}{u_{2}}\right)=\frac{D\left(r_{1} r_{2}\right)}{u_{1} u_{2}}-\frac{r_{1} r_{2} D\left(u_{1} u_{2}\right)}{\left(u_{1} u_{2}\right)^{2}}=\frac{r_{1} D\left(r_{2}\right)+D\left(r_{1}\right) r_{2}}{u_{1} u_{2}}-\frac{r_{1} r_{2}\left(u_{1} D\left(u_{2}\right)+D\left(u_{1}\right) u_{2}\right)}{\left(u_{1} u_{2}\right)^{2}}= \\
=\frac{r_{1}}{u_{1}}\left(\frac{D\left(r_{2}\right)}{u_{2}}-\frac{r_{2} D\left(u_{2}\right)}{u_{2}^{2}}\right)-\frac{r_{2}}{u_{2}}\left(\frac{D\left(r_{1}\right)}{u_{1}}+\frac{r_{1} D\left(u_{1}\right)}{u_{1}^{2}}\right)=\frac{r_{1}}{u_{1}} D^{\prime}\left(\frac{r_{2}}{u_{2}}\right)+\frac{r_{2}}{u_{2}} D^{\prime}\left(\frac{r_{1}}{u_{1}}\right) .
\end{gathered}
$$

Mostrar que $d^{\prime}$ é de fato uma derivação é análogo e a unicidade segue, em ambos os casos, da regra de Leibniz quando calculamos $D^{\prime}\left(\frac{r}{u}\right)=D^{\prime}\left(\frac{r}{1} \frac{1}{u}\right)$.

Exemplo 14. Como consequencia dessa proposição temos que $\operatorname{Der}(\mathbb{Q})=0$, pois dado $z \in \mathbb{Z}$, $d(z)= \pm d(\underbrace{1+1+\cdots+1}_{|\mathrm{z}| \text { vezes }})=0$ e então $d\left(\frac{a}{b}\right)=\frac{d(a) b-a d(b)}{b^{2}}=0$

\subsubsection{Derivações em corpos}

Sejam $k \subseteq F \subseteq L$ corpos. Tome $d \in \operatorname{Der}_{k}(F, L)$ e seja $X$ uma indeterminada e $g(X) \in K[X]$. Denotamos por $g^{d}(X)$ o polinômio obtido aplicando $d$ em cada um dos coeficientes de $g(X)$ e denotamos por $g^{\prime}(X)$ a derivada usual de $g(X)$ em relação a $X$.

Verificações simples nos mostram que os morfismos $g(X) \rightarrow g^{d}(X)$ e $g(X) \rightarrow g^{\prime}(X)$ são $k$-derivações de $F(X)$ em si mesmo e também que a aplicação $g(x) \rightarrow g^{d}(X)+p(X) g^{\prime}(X)$ é uma $k$-derivação de $F(X)$ para todo polinômio $p(X) \in F[X]$. Note que nesse caso $X \mapsto p(X)$.

Proposição 15. Sejam $k \subseteq F \subseteq L \subseteq M$ corpos, com $L$ sendo uma extensão separável de $F$. Tome $d \in \operatorname{Der}_{k}(F, M)$, então $d$ pode ser estendida a uma derivação $\bar{d} \in \operatorname{Der}_{k}(L, M)$. Se $L$ é algébrico sobre $F$, então essa extensão de $d$ é única e se $a \in L$, com polinômio minimal $g(X) \in F[X]$, então $\bar{d}(a)$ é o único elemento de $M$ que satisfaz

$$
g^{d}(a)+\bar{d}(a) g^{\prime}(a)=0
$$

Demonstração: A demonstração pode ser encontrada na referência (ZARISKI; SAMUEL, 1958, p.-122) teorema 9, et seq.

Corolário 16. Seja $F$ uma extensão algébrica e separável de um corpo $k$, então $\operatorname{Der}_{k}(F)=0$.

Demonstração: Seja $d \in \operatorname{Der}_{k}(F)$, então tanto $d$ quanto 0 estendem a derivação 0 de $k$ em $k$, mas pela proposição anterior a extensão é única, $\operatorname{logo} d=0$.

\subsubsection{Geometria Algébrica}

Ao longo dessa sessão enunciarei vários resultados bem conhecidos de geometria algébrica, sem suas demonstrações, que podem ser encontradas em (HARTSHORNE, 1977). 
Seja $k$ um corpo, defina o espaço afim $n$-dimensional, denotado por $\mathbb{A}_{k}^{n}$, como $\mathbb{A}_{k}^{n}=\left\{P=\left(a_{1}, a_{2}, \cdots, a_{n}\right) ; a_{i} \in k\right.$ para $\left.i=1, \cdots, n\right\}$. Seja $R=k\left[X_{1}, \cdots, X_{n}\right]$ o anel de polinômios em $n$ variáveis sobre $k$. Dado $T$ um subconjunto de $R$, definimos o conjunto dos zeros de $T$ em $\mathbb{A}_{k}^{n}$ por

$$
V(T)=\left\{P \in \mathbb{A}_{k}^{n} ; f(P)=0 \text { para todo } f \in T\right\} .
$$

Note que se $J \subseteq R$ é um ideal gerado pelos elementos de $T$, então $V(T)=V(J)$. Além disso, como $R$ é noetheriano, temos que $V(T)$ pode ser visto como o conjunto dos zeros de um número finito de polinômios, os geradores do ideal $J$.

Definição 17. Um conjunto $Y \subseteq \mathbb{A}_{k}^{n}$ é um conjunto algébrico se existe $T \subseteq R$ tal que $Y=V(T)$.

Tome agora um subconjunto $Y$ de $\mathbb{A}_{k}^{n}$. Definimos o ideal de $Y$ em $R$ por

$$
I(Y)=\{f \in R ; f(P)=0 \text { para todo } P \in Y\}
$$

Definição 18. Um conjunto algébrico $Y \subseteq \mathbb{A}_{k}^{n}$ é dito irredutível se não pode ser expresso como a união de dois conjuntos algébricos próprios não vazios, isto é, se $Y_{1}, Y_{2}$ são conjuntos algébricos próprios e $Y=Y_{1} \cup Y_{2}$, então $Y_{1}=\varnothing$ ou $Y_{2}=\varnothing$. Caso contrário $Y$ é dito redutível.

Proposição 19. Temos as seguintes relações entre as funções $T \rightarrow V(T)$ e $Y \rightarrow I(Y)$ :

1. Se $T_{1} \subseteq T_{2} \subseteq R$, então $V\left(T_{2}\right) \subseteq V\left(T_{1}\right)$

2. Se $Y_{1} \subseteq Y_{2} \subseteq \mathbb{A}_{k}^{n}$, então $I\left(Y_{2}\right) \subseteq I\left(Y_{1}\right)$.

3. Dados $Y_{1}, Y_{2} \subseteq \mathbb{A}_{k}^{n}$, temos que $I\left(Y_{1} \cup Y_{2}\right)=I\left(Y_{1}\right) \cap I\left(Y_{2}\right)$.

4. Se $Y \subseteq \mathbb{A}_{k}^{n}$ é um conjunto algébrico, então $V(I(Y))=Y$.

Teorema 20 (Hilbert's Nullstellensatz ). Seja $k$ um corpo algebricamente fechado, $I$ um ideal de $R=k\left[X_{1}, \cdots, X_{n}\right]$ e $f \in R$ um polinômio que se anula em todos os pontos de $V(I)$. Então $f^{r} \in I(V)$ para algum inteiro positivo $r$.

Corolário 21. Temos uma correspondência que inverte inclusões entre os conjuntos algébricos em $\mathbb{A}_{k}^{n}$ e ideais radicais de $R$. Além disso, um conjunto algébrico é irredutível se, e somente se, seu ideal é primo.

Um ideal maximal em $R$ corresponde a um conjunto algébrico irredutível minimal em $\mathbb{A}_{k}^{n}$, neste caso, um ponto $P=\left(a_{1}, \cdots, a_{n}\right)$. Então todo ideal maximal de $R$ é da forma $\left(x_{1}-a_{1}, \cdots, x_{n}-a_{n}\right)$ e como um ideal maximal é radical, todo ponto corresponde a um ideal dessa forma.

Proposição 22. Seja $V \subseteq \mathbb{A}_{k}^{n}$ um conjunto algébrico. Então $V$ pode ser expresso de maneira única como uma união finita de subconjuntos algébricos próprios distintos $V=V_{1} \cup \cdots \cup V_{m}$ e se $P_{i}=I\left(V_{i}\right)$, então $I(V)=P_{1} \cap \cdots \cap P_{m}$. 
Tais $V_{i}$ são conhecidos como componentes irredutíveis de $V$ e os ideais primos $P_{i}$ são os primos minimais de $I(V)$.

Definição 23. Seja $V \subseteq \mathbb{A}_{k}^{n}$ um conjunto algébrico. Definimos o anel de coordenadas afins de $V$, denotado por $A(V)$, por $A(V)=R / I(V)$.

Note que se $B$ é uma álgebra reduzida finitamente gerada sobre $k$ então $B$ é o anel de coordenadas de um conjunto algébrico, pois $B=k\left[X_{1}, \cdots, X_{n}\right] /\left(f_{1}, \cdots, f_{m}\right)$ onde $m, n$ são inteiros não negativos e $\left(f_{1}, \cdots, f_{m}\right)$ um ideal radical, $\log$ o $B$ é o anel de coordenadas do conjunto algébrico $V\left(f_{1}, \cdots, f_{m}\right)$ em $\mathbb{A}_{k}^{n}$. Além disso, pelo teorema 20 temos uma correspondência entre os conjuntos algébricos irredutíveis e álgebras finitamente geradas que são um domínio.

Seja $V=V(J)$ um conjunto algébrico dado pelos zeros do ideal radical $J \subseteq R$. Temos uma correspondência entre os ideais de $R$ que contêm o ideal $J$ e os ideais de $R / J=A(V)$. Consequentemente temos uma correspondência entre os subconjuntos algébricos de $V$ e os ideais radicais de $A(V)$.

Definição 24. Sejam $V \in \mathbb{A}_{k}^{n}$ um conjunto algébrico, $A(V)=R / I$ seu anel de coordenadas com $I=\left(f_{1}, \cdots, f_{m}\right)$ e $P=\left(a_{1}, \ldots, a_{n}\right) \in V$ um ponto. Temos que $P$ corresponde em $R$ ao ideal gerado por $I_{P}=\left\langle X_{1}-a_{1}, \ldots, X_{n}-a_{n}\right\rangle$. Tome $M_{P}=I_{p} / I$ o ideal maximal de $A(V)$ correspondente ao ponto $P$.

Um vetor $\left(t_{1}, \ldots, t_{n}\right) \in\left(A(V) / M_{P}\right)^{n}$ (que é um espaço vetorial $n$-dimensional, sobre o corpo $\left.A(V) / M_{P}\right)$ é tangente a $V$ em $P$ se

$$
\sum_{i=1}^{n} t_{j} \cdot \frac{\partial f_{j}(P)}{\partial X_{j}}=0 \quad \text { para todo } 1 \leq j \leq m
$$

O conjunto $T_{P} V$ dos vetores tangentes a $V$ em $P$ é um espaço vetorial sobre $k$ que independe da escolha de geradores de $I$.

Mantendo a notação da definição anterior e analisando o teorema 7, segue a seguinte proposição:

Proposição 25. Para cada $P \in V$, seja $\phi_{P}: A(V) \rightarrow A(V) / M_{P}$ a projeção natural. Então $T_{P} V \simeq$ $\operatorname{Der}_{k}\left(A(V), A(V) / M_{p}, \phi_{P}\right)$.

Corolário 26. Cada elemento de $\operatorname{Der}_{k}(A(V))$ induz um vetor tangente à $V$ em cada ponto de $V$.

Demonstração: Sejam $d \in \operatorname{Der}_{k}(A(V))$ e $P$ um ponto de $V$. Então $\phi_{P} \circ d \in \operatorname{Der}_{k}\left(A(V), A(V) / M_{P}, \phi_{P}\right)$ que é isomorfo a $T_{P} V$ pela proposição anterior. Portanto $d$ induz um vetor tangente a $V$ em $P$. 
CAPÍTULO

\section{3}

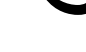

\section{D-SIMPLICIDADE}

\section{1 d-simplicidade}

A seguir relembraremos a definição de $d$-simplicidade.

Definição 27. Seja $A$ um anel e $d \in \operatorname{Der}(A)$. Um ideal $I \subseteq A$ é um $d$-ideal se $d(I) \subseteq I$. $A$ é $d$-simples caso possua apenas (0) e $A$ como $d$-ideais.

Exemplo 28. Seja $K$ um corpo, considere $A=K[X]$ e $B=K[X, Y]$. Tome as derivações $d_{1}=\frac{\partial}{\partial X}$ e $d_{2}=X \frac{\partial}{\partial X}$

- A é $d_{1}$ - simples, pois $A$ é domínio de ideais principais, portanto todo ideal não nulo pode ser escrito na forma $I=\langle f\rangle, f \in A \backslash\{0\}$. Se $\operatorname{deg}(f)=n>0$ então $\operatorname{deg}\left(d_{1}(f)\right)=n-1$ e portanto $d_{1}(f) \notin I$. Caso $\operatorname{deg}(f)=0$ temos $I=A$ e portanto $A$ e $\langle 0\rangle$ são os únicos $d_{1}$-ideais.

- $A$ não é $d_{2}$-simples. De fato $I=\langle X\rangle$ é um $d_{2}$-ideal, pois se $f \in I$ então $f=X g, g \in A$ e $d_{2}(f)=X g+X d_{2}(g)=X\left(g+d_{2}(g)\right) \in I$.

- $B$ não é $d_{1}$-simples, de fato $\langle Y\rangle$ é um $d_{1}$ - ideal.

- $B$ é $d$-simples com a derivação $d=\frac{\partial}{\partial X}+(1+X Y) \frac{\partial}{\partial Y}$. Provaremos esse fato mais tarde, usando o teorema de Shamsuddin.

- $K[X, Y, Z]$ não é $d$-simples com $d=\frac{\partial}{\partial X}+(1+X Y) \frac{\partial}{\partial Y}+(1+X Z) \frac{\partial}{\partial Z}$. De fato $(Y-Z)$ é um $d$ - ideal, pois $d(f(Y-Z))=(Y-Z) d(f)+f d(Y-Z)=(Y-Z)(d(f)+X f)$ para todo $f \in K[X, Y, Z]$

Lema 29. Seja $A$ um anel e $I \subseteq A$ um ideal com geradores $I=\left(f_{\alpha}\right)_{\alpha \in \Lambda}$, então $I$ é um $d$-ideal se, e somente se, $d\left(f_{\alpha}\right) \in I$ para todo $\alpha \in \Lambda$. 
Demonstração: Se $I$ é um $d$-ideal, então $d\left(f_{\alpha}\right) \in I$ para todo $\alpha \in \Lambda$.

Suponha agora que $d\left(f_{\alpha}\right) \in I$ para todo $\alpha \in \Lambda$. Dado um elemento $f \in I$ qualquer, temos que $f=\sum_{i=0}^{n} a_{i} f_{\alpha_{i}}$ para algum $n \in \mathbb{N}, a_{i} \in A$ e índices $\alpha_{i} \in \Lambda$ convenientes. $\operatorname{Logo} d(f)=$ $d\left(\sum_{i=0}^{n} a_{i} f_{\alpha_{i}}\right)=\sum_{i=0}^{n} \underbrace{d\left(a_{i}\right) f_{\alpha_{i}}+a_{i} d\left(f_{\alpha_{i}}\right)}_{\in I}$, portanto $d(f) \in I$ e $I$ é um $d$-ideal.

Observação 30. Sejam $R$ um anel e $I \subseteq R$ um ideal. A observação 5 nos diz que as derivações de $R / I$ em $R / I$ estão em correspondência um a um com as derivações $d: R \rightarrow R$ tais que $I$ é um $d$-ideal.

\subsection{Lema de Zariski}

Teorema 31 (Lema de Zariski). Seja $A$ um anel local completo. Suponha que exista uma derivação $d \in \operatorname{Der}(A)$ tal que $d(x)$ é uma unidade em $A$, mas $x$ não o é. Então $A$ contém um subanel $B$ tal que

1. $d$ é identicamente $0 \mathrm{em} B$.

2. $x$ é analiticamente independente sobre $B$.

3. $A=B[[x]]$.

Demonstração: Como $d(x)$ é uma unidade em $A$, podemos assumir que $d(x)=1$, pois $d(x)^{-1} d \in \operatorname{Der}(A)$. Para $n>0 \in \mathbb{N}$ considere $d^{n}(a)$ como $d$ aplicada $n$ vezes sucessivamente em $a$ e $d^{0}(a)=a$ para todo $a \in A$. Vamos fazer o uso de alguns fatos:

1. A aplicação $E: A \rightarrow A$, definida em cada elemento $a \in A$ por $E(a)=\sum_{i=0}^{\infty}(-1)^{n} \frac{x^{i}}{i !} d^{i}(a)$, é um endomorfismo de $A$. Note que $E$ está bem definida já que $A$ é anel local completo e $x$ não é uma unidade de $A$.

2. Se $B=E(A)$ e $b \in B$, então $b=a-x \cdot d(a)+\frac{x^{2}}{2 !} \cdot d^{2}(a)-\cdots$ para algum $a \in A$ e $d(b)=$ $d(a)-d(a)-x d^{2}(a)+x d^{2}(a)+\cdots=0$. Portanto $d(B)=0$.

3. Como $E(x)=0$ então $E(a x)=0$ para todo $a \in A$ e portanto $A x \subseteq \operatorname{Ker}(E)$. Agora considere $a \in \operatorname{Ker}(E)$, então $E(a)=0=a-x d(a)+\frac{x^{2}}{2 !} d^{2}(a)-\cdots$ e então $a=x\left(d(a)-\frac{x}{2 !} d^{2}(a)+\right.$ $\cdots)$, portanto $a \in A x$. Logo $A x=\operatorname{Ker}(E)$.

4. Se $b \in B$, como $d(b)=0$, temos que $E(b)=b$. E portanto $E^{2}=E$.

5. Seja $c \in B \cap A x$, então $E(c)=c$, pois $c \in B$, mas $c \in A x=\operatorname{Ker}(E)$ e então $E(c)=0$. Portanto $A x \cap B=0$. 
Dado $B=E(A)$, subanel de $A$ que construímos anteriormente, vamos provar que $x$ é analiticamente independente sobre $B$. Suponha que exista um conjunto de índices $\left\{b_{i} \in B\right\}$ com $b_{0} \neq 0$ tal que

$$
\left(\sum_{i=0}^{\infty} b_{i} x^{i}\right) x^{s}=0 \text { para algum inteiro } s \geq 0 .
$$

Aplicando $d^{s}$ a equação acima e lembrando que $d(B)=0$ obtemos

$$
\begin{gathered}
\sum_{i=0}^{\infty} \frac{(i+s) !}{i !} b_{i} x^{i}=0 \text { e então } \\
s ! b_{0}=-x \sum_{i=1}^{\infty} \frac{(i+s) !}{i !} b_{i} x^{i-1},
\end{gathered}
$$

portanto $b_{0} \in B \cap A x=0$, contradizendo que $b_{0} \neq 0$. Portanto $x$ é analiticamente independente sobre $B$.

Vamos mostrar agora que todo elemento de $A$ pode ser expresso unicamente como um elemento de $B[[x]]$.

Para cada $a \in A, E(a-E(a))=E(a)-E^{2}(a)=E(a)-E(a)=0$, portanto $a-E(a) \in$ $\operatorname{Ker}(E)=A x$ e então podemos escrever $a=b_{0}+a_{1} x$, onde $b_{0}=E(a) \in B$ e $a_{1} \in A$. Similarmente $a_{1}$ pode ser expresso como $b_{1}+a_{2} x$, onde $b_{1}=E\left(a_{1}\right)$ e $a_{2} \in A$. Prosseguindo por indução com esses passos vamos obter uma expressão

$$
a=b_{0}+b_{1} x+b_{2} x^{2}+\cdots .
$$

Essa expressão é única pois $x$ é analiticamente independente sobre $B$. Portanto $A=B[[x]]$.

Corolário 32. Seja $K$ um corpo e $R=K\left[\left[X_{1}, \ldots X_{n}\right]\right]$ com $n \geq 2$. Então $R$ não é $d$-simples para qualquer derivação $d \in \operatorname{Der}_{K}(R)$.

Demonstração: $R$ é um anel local completo. Suponha que $R$ é $d$-simples para alguma $d \in \operatorname{Der}_{K}(R)$. Seja $m$ o único ideal maximal de $R$, como ele não é um $d$-ideal, existe $x \in m$ tal que $d(x) \notin m$. Logo $d(x)$ é uma unidade, mas $x$ não o é. Pelo teorema anterior $R=B[[x]]$, com $d(B)=0$. Note que $B \neq K$, pois $n \geq 2$. De fato, se $B=K$ então $n=\operatorname{dim}(R)=\operatorname{dim}(K[[x]])=1$, contradição. Claramente qualquer ideal próprio de $R$ gerado por elementos de $B$ é um $d$-ideal. Contradição.

Proposição 33. Seja $R$ um anel noetheriano e $d \in \operatorname{Der}(R)$. Se $I$ é um $d$-ideal de $R$ então os primos minimais de $I$ também são $d$-ideais.

Demonstração: Temos que $d(I) \subseteq I$ então, pela proposição $4, d$ induz uma derivação, que também denotaremos por $d$, no anel quociente $S=R / I$. Seja $P \subseteq S$ um ideal primo minimal com $d(P) \nsubseteq P$. Temos pela proposição 13 que $d$ pode ser estendida unicamente a uma derivação, 
que também denotaremos por $d$, em $S_{P}$. Então $d\left(P_{P}\right) \nsubseteq P_{P}$ e portanto $d$ leva uma não-unidade de $S_{P}$ em uma unidade. Como $P$ é minimal, o anel local $S_{P}$ é zero dimensional, além disso como $S_{P}$ é noetheriano temos que ele também é artiniano. Pelo teorema 7.3.1 da referência (BORGES; TENGAN, 2015, p.203) temos que $P_{P}$ é nilpotente, portanto $S_{P}$ é completo (toda sequencia de Cauchy se torna eventualmente constante). Pelo teorema anterior existem $x \in S_{P}$ e um subanel $B \subseteq S_{P}$ tais que $S_{P}=B[[x]]$, mas $\operatorname{dim}(B[[x]]) \geq 1$, contradição. Portanto $d(P) \subseteq P$, para todo primo minimal $P$ de $S$. Como todo primo minimal de $S$ corresponde a um ideal primo minimal sobre $I$ em $R$, o resultado segue pela observação 5 .

Corolário 34. Seja $R$ um anel noetheriano. Se $R$ é $d$-simples para alguma derivação $d \in \operatorname{Der}(R)$, então $R$ é um domínio.

Demonstração: Pela proposição anterior, todos os primos minimais de $R$ são $d$-ideais para toda $d \in \operatorname{Der}(R)$, pois (0) é um $d$-ideal. Consequentemente se $R$ é $d$-simples o único ideal primo minimal é $(0)$. Portanto $R$ é um domínio.

Corolário 35. Seja $d \in \operatorname{Der}(R)$, então $R$ é $d$-simples se e somente se $R$ não contém $d$-ideais primos não nulos.

Demonstração: Se $R$ não contém $d$-ideais primos não nulos, então pela proposição anterior, $R$ não contém $d$-ideais. A recíproca é direta pela definição.

Observação 36. Geometricamente, os ideias primos minimais do anel de coordenadas $R$ de um conjunto algébrico $V$ definido sobre um corpo $K$, são os ideais primos correspondentes às componentes irredutíveis de V. Digamos que $V=V_{1} \cup V_{2} \cup \cdots \cup V_{n}$, com $V_{i}$ irredutível. Pelo corolário 26, toda $K$-derivação de $R$ induz um campo vetorial tangente em $V$. Então, pela observação 5, uma derivação $d \in \operatorname{Der}(R)$ induz uma derivação no anel de coordenadas de cada $V_{i}$ e consequentemente dá origem a uma campo vetorial que é tangente a $V$ e a cada uma de suas componentes irredutíveis.
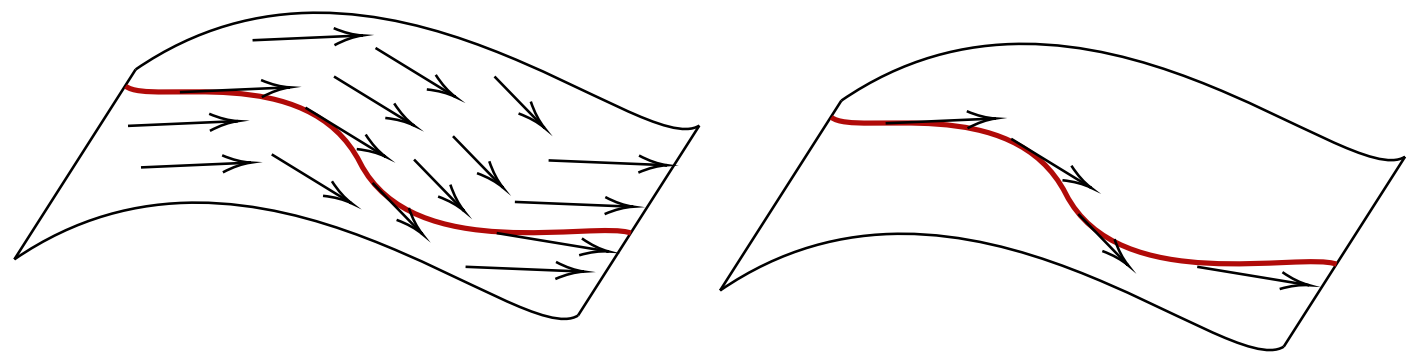

Figura 1 - Campo vetorial restrito à subvariedade. 


\section{3 d-simplicidade em extensões inteiras}

Teorema 37. Sejam $R \subseteq T$ anéis comutativos (contendo $\mathbb{Q}$ ) com $T$ inteiro sobre $R$ e suponha que $d \in \operatorname{Der}(T)$ é tal que $d(R) \subseteq R$. Seja $P$ um $d$-ideal primo de $R$. Então os ideais primos de $T$ que estão sobre $P$ são também $d$-ideais.

Demonstração: Suponha que $Q \cap R=P$ onde $Q$ é um ideal primo de $T$. Seja $x \in Q$, então $x$ satisfaz uma relação polinomial

$$
F(x)=x^{n}+r_{n-1} x^{n-1}+\cdots+r_{1} x+r_{0}=0 \operatorname{com} r_{i} \in R,
$$

então $r_{0}=-x\left(x^{n-1}+\cdots r_{1}\right) \in Q \cap R=P$, mas $d(P) \subseteq P$ implica que $d\left(r_{0}\right) \in P$.

Temos que $d(F(x))=x\left(n \cdot x^{n-2} \cdot d(x)+\cdots+r_{1}\right)+r_{1} \cdot d(x)+d\left(r_{0}\right)=0$ e como $x$ e $d\left(r_{0}\right) \in Q$ segue que $r_{1} . d(x) \in Q$. Se $r_{1} \notin P$ então $d(x) \in Q$, o que nos dá o desejado. Suponha agora que $r_{1} \in P$, então $d\left(r_{1}\right) \in P$ e $0=d^{2}(F(x))=x(\cdots)+\left(2 d(x) r_{2}+d\left(r_{1}\right)\right) d(x)+d\left(r_{1}\right) d(x)+r_{1} d^{2}(x)+d^{2}\left(r_{0}\right)$ e teremos então que $2 d(x)^{2} r_{2} \in Q$. Em geral, se $r_{1}, \cdots, r_{i-1} \in P$ então $d^{i}(F(x))=0$ implica que $i ! d(x)^{i} r_{i+1} \in Q$. Se algum $r_{i} \notin P$ segue que $d(x) \in Q$, mas se todo $r_{i} \in P$ então $d^{n}(F(x))=0$ implica que $n ! d(x)^{n} \in Q$. Portanto $d(x) \in Q$ para todo $x \in Q$ e $Q$ é um $d$-ideal.

Teorema 38. Seja $K$ um corpo e $\bar{K}$ seu fecho algébrico. Seja $R=K\left[x_{1}, \cdots, x_{n}\right]$ uma $K$-álgebra finitamente gerada. Então $T=\bar{K}\left[x_{1}, \cdots, x_{n}\right]$ é uma extensão inteira de $R$.

Demonstração: Seja $0 \neq f \in T$ e $\alpha_{1}, \cdots, \alpha_{s} \in \bar{K}$ os coeficientes não nulos de $f$. Considere $S=$ $K\left(\alpha_{1}, \cdots, \alpha_{s}\right)\left[x_{1}, \cdots, x_{n}\right]$. Como $K\left(\alpha_{1}, \cdots, \alpha_{n}\right)$ é uma extensão algébrica de $K, S$ é finitamente gerado como $R$-módulo e então é inteiro sobre $R$. Como $f \in S$ segue que $f$ é inteiro sobre $R$.

Teorema 39. Sejam $K$ um corpo e $\bar{K}$ seu fecho algébrico. Sejam $R=K\left[x_{1}, \cdots, x_{n}\right]$ e $T=\bar{K}\left[x_{1}, \cdots, x_{n}\right]$ domínios finitamente gerados. Seja $d \in \operatorname{Der}_{K}(R)$, então $d$ pode ser estendida a uma derivação $\bar{d} \in \operatorname{Der}_{\bar{K}}(T)$ definindo $\bar{d}\left(x_{i}\right)=d\left(x_{i}\right)$ para cada $i=1, \cdots, n$. Além disso $R$ é $d$-simples se e somente se $T$ é $\bar{d}$-simples

Demonstração: Seja $R=K[\underline{X}] / I$ para algum ideal $I \subseteq K[X]$, então $T=\bar{K}[\underline{X}] / I \bar{K}[\underline{X}]$. Pela proposição $4, d$ pode ser "levantada"a uma derivação $D \in \overline{D e r}_{K}(K[\underline{X}])$ satisfazendo $D(I) \subseteq$ I. Além disso, $D$ pode ser estendida a uma $\bar{K}$-derivação $\bar{D}$ de $\bar{K}[\underline{X}]$ simplesmente tomando $\bar{D}\left(X_{i}\right)=D\left(X_{i}\right)$ para cada $i$. Temos que $\bar{D}(I T) \subseteq I T$ e então $\bar{D}$ induz uma derivação $\bar{d} \in \operatorname{Der}_{\bar{K}}(T)$ e $\bar{d}\left(x_{i}\right)=d\left(x_{i}\right)$.

Como $R \subseteq T$ e $\bar{d}$ se restringe a $d$ em $R$ falta apenas mostrar que $R$ é $\bar{d}$-simples se e somente se $T$ é $\bar{d}$-simples.

Pelo teorema anterior, $T$ é uma extensão inteira de $R$. Suponha que $Q$ é um $\bar{d}$-ideal não nulo de $T$, então $Q \cap R$ é um $\bar{d}$-ideal não nulo de $R$, já que a extensão $T / R$ é inteira. Portanto $Q \cap R=R$ o que implica $Q=T$. Então, se $R$ é $d$-simples, $T$ também é $\bar{d}$-simples.

A volta segue diretamente do teorema 37. 


\section{4 d-ideais maximais}

Seja $R=k\left[x_{1}, \cdots, x_{n}\right]$ o anel de coordenadas de uma variedade $V$ definida sobre $k$ e seja $d \in$ $\operatorname{Der}_{k}(R)$, então $d$ induz um campo vetorial tangente em $V$. Seja $\alpha \in V$ um ponto e $M \subseteq R$ o ideal maximal correspondente. Suponha que o vetor tangente a $V$ em $\alpha$ seja nulo, isto é $\left(d\left(x_{1}\right), \cdots, d\left(x_{n}\right)\right) \equiv(0,0, \cdots, 0) \bmod M$. Então $d\left(x_{i}\right) \in M$ para cada $i$ e portanto $d(R) \subseteq M$. Em particular $d(M) \subseteq M$. Isto é, se o campo vetorial induzido por $d$ se anula em algum ponto então $d$ deixa o ideal maximal correspondente invariante. Note também que se $d(R) \subseteq M$ então o campo vetorial tangente se anula em $\alpha$. Vamos usar o seguinte resultado para ter a recíproca completa.

Teorema 40. Seja $K$ um corpo e $R$ uma $K$-álgebra. Suponha que M é um ideal maximal de $R$ tal que $R / M$ é uma extensão algébrica de $K$. Seja $d \in \operatorname{Der}(R)$, então $d(M) \subseteq M$ se e somente se $d(R) \subseteq M$.

Demonstração: Suponha que $d(M) \subseteq M$, então $d$ induz uma $K$-derivação $d^{\prime}: R / M \rightarrow R / M$ dada por $d^{\prime}(r+M)=d(r)+M$ para cada $r \in R$. Pelo corolário $16, \operatorname{Der}_{K}(R / M)=0$. Então $d^{\prime}=0$ e portanto $d(r) \in M$ para todo $r \in R, \operatorname{logo} d(R) \subseteq M$.

A reciproca é clara.

Corolário 41. Seja $R=K\left[x_{1}, \cdots, x_{n}\right]$ uma $K$-álgebra finitamente gerada e seja $d \in \operatorname{Der}_{k}(R)$. Então $R$ não tem $d$-ideais maximais se e somente se $d\left(x_{1}\right) R+\cdots+d\left(x_{n}\right) R=R$.

Demonstração: Como $R$ é uma $K$-álgebra finitamente gerada, pelo Teorema dos Zeros de Hilbert, $R / M$ é uma extensão algébrica de $K$ pelo corolário 5.24 da referencia (ATIYAH; MACDONALD, 1969 , p. 67). Logo, pelo teorema anterior, $R$ não tem $d$-ideias maximais se e somente se $d(R) \not \subset M$, para todo ideal maximal $M$. E isso ocorre se e somente se $d\left(x_{1}\right) R+\cdots+d\left(x_{n}\right) R=R$ já que $d(R)=d\left(x_{1}\right) R+\cdots+d\left(x_{n}\right) R$.

Corolário 42. Seja $R=K\left[x_{1}, \ldots, x_{n}\right]$ uma $K$-álgebra finitamente gerada. Então $R$ admite uma derivação tal que nenhum ideal maximal de $R$ é um $d$-ideal se e somente se $\Omega_{K}(R)$ admite um somando direto livre de posto 1 .

Demonstração: Suponha que $d \in \operatorname{Der}_{K}(R)$ e que $R$ não tem $d$-ideais maximais. Então $\sum_{i=1}^{n} r_{i} d\left(x_{i}\right)=1$, para alguns $r_{i} \in R$. Seja $d_{K}^{R}$ a $K$-derivação universal de $R$ em $\Omega_{K}(R)$. Pela propriedade universal existe um único $h \in \operatorname{Hom}_{R}\left(\Omega_{K}(R), R\right)$ tal que $h d_{K}^{R}=d$. Seja $\omega=\sum_{i=1}^{n} r_{i} d_{K}^{R}\left(x_{i}\right)$. Note que $\omega \in \Omega_{K}(R)$ e

$$
h(\omega)=\sum_{i=1}^{n} r_{i} h d_{k}^{R}\left(x_{i}\right)=\sum_{i=1}^{n} r_{i} d\left(x_{i}\right)=1 .
$$

Portanto $h$ é sobrejetora e a sequencia exata curta

$$
0 \longrightarrow \operatorname{Ker}(h) \longrightarrow \Omega_{K}(R) \stackrel{h}{\longrightarrow} R \longrightarrow 0
$$

cinde, o que nos dá que $\Omega_{k}(R) \simeq R \oplus \operatorname{Ker}(h)$.

Para a volta suponha agora que $\Omega_{K}(R)=R \oplus S$ para algum $R$-módulo $S$. Então existe um 
homomorfismo sobrejetivo $h: \Omega_{K}(R) \rightarrow R$ e portanto um elemento $\omega \in \Omega_{K}(R)$ tal que $h(\omega)=1$. Agora $d_{K}^{R}\left(x_{1}\right), \ldots, d_{K}^{R}\left(x_{n}\right)$ são geradores de $\Omega_{K}(R)$ como $R$-módulo (note que isso segue da definição de $\Omega_{k}(R)$ e do fato que $d_{k}^{R} \equiv 0$ em $k$ ) e então podemos escrever

$$
\omega=\sum_{i=1}^{n} r_{i} d_{K}^{R}\left(x_{i}\right) \quad r_{i} \in R .
$$

Seja $d=h d_{K}^{R}$, então

$$
\sum_{i=1}^{n} r_{i} d\left(x_{i}\right)=h\left(\sum_{i=1}^{n} r_{i} d_{K}^{R}\left(x_{i}\right)\right)=h(\omega)=1 .
$$

Pelo corolário anterior, $R$ não possui $d$-ideais maximais.

\subsection{Adjuntando uma variável}

Seja $R$ um domínio (contendo $\mathbb{Q}$ ) e $X$ uma indeterminada. Seja $d \in \operatorname{Der}(R[X])$ tal que $d(R) \subseteq R$ e suponha que $d(X)=f(X)$. Então

$$
d(g(X))=g^{d}(X)+f(X) \frac{\partial g(X)}{\partial X} \text { para todo } g \in R[X]
$$

onde $g^{d}(X)$ é o polinômio obtido aplicando $d$ a cada um dos coeficientes de $g(X)$. Analogamente, suponha que $d \in \operatorname{Der}(R)$ e seja $f \in R[X]$. Então podemos construir uma derivação de $R[X]$, também denotada por $d$, tal que $d(X)=f(X)$ e cuja restrição em $R$ coincide com a derivação $d$ inicial, definindo $d(g(X))=g^{d}(X)+f(X) \frac{\partial g(X)}{\partial X}$.

Sejam $K$ o corpo quociente de $R$ e $d \in \operatorname{Der}(R[X])$ tal que $d(R) \subseteq R$. Então pela proposição 13, $d$ pode ser estendida unicamente a uma derivação de $K[X]$ tal que $d(K) \subseteq K$. Note que os $d$-ideais primos de $K[X]$ estão em correspondência um a um com os $d$-ideais primos de $R[X]$ que não intersectam $R \backslash\{0\}$, pois $K$ é a localização de $R$ em $R \backslash\{0\}$.

Proposição 43. Seja $K$ um corpo (contendo $\mathbb{Q}$ ) e $\bar{K}$ seu fecho algébrico. Seja $d \in \operatorname{Der}(K[X])$ tal que $d(K) \subseteq K$ e suponha que $d(X)=f(X)$. Então $K[X]$ é $d$-simples se e somente se $d(\alpha) \neq f(\alpha)$ para todo $\alpha \in \bar{K}$.

Demonstração: $K[X]$ é um domínio de ideais principais, logo todo ideal primo pode ser gerado por um polinômio mônico irredutível. Seja $q(X)$ um polinômio mônico irredutível e $\alpha \in \bar{K}$ uma raiz de $q(X)$. Então $q(X)$ gera um $d$-ideal de $K[X]$ se e somente se

$$
d(q(X))=q^{d}(X)+f(X) \frac{\partial q(X)}{\partial X}=p(X) q(X), \text { para algum } p(X) \in K[X] .
$$

Isso acontece se e somente se

$$
q^{d}(\alpha)+f(\alpha) \frac{\partial q(\alpha)}{\partial X}=p(\alpha) q(\alpha)=0
$$

e pela proposição 15 , isso é verdade se e somente se $d(\alpha)=f(\alpha)$. 
Corolário 44. Sejam $R$ um domínio, $K$ seu corpo quociente e $\bar{K}$ o fecho algébrico de $K$. Sejam $X$ uma indeterminada, $d \in \operatorname{Der}(R[X])$ tal que $d(R) \subseteq R$ e $d(X)=f(X)$. Assuma que $R$ é $d$-simples. Então $R[X]$ é $d$-simples se e somente se $d(\alpha) \neq f(\alpha)$ para todo $\alpha \in \bar{K}$.

Demonstração: Temos que os $d$-ideais primos de $R[X]$ estão em bijeção com os $d$-ideais primos de $K[X]$. Então $R[X]$ é $d$-simples se e somente se $K[X]$ é $d$-simples e o resultado segue da preposição anterior.

Teorema 45 (Shamsuddin). Sejam $R$ um domínio e $K$ seu corpo quociente. Sejam $X$ uma indeterminada, $d \in \operatorname{Der}(R[X])$ tal que $d(R) \subseteq R$ e $d(X)=f(X)$ com $\operatorname{deg}(f) \leq 1$. Assuma que $R$ é $d$-simples. Então $R[X]$ é $d$-simples se $d(r) \neq f(r)$ para todo $r \in R$.

Demonstração: Seja $f(X)=f_{0}+f_{1} X, f_{i} \in R$. Suponha que $d(r) \neq f_{0}+f_{1} r$ para todo $r \in R$. Suponha que $J \subsetneq R[X]$ é um $d$-ideal próprio. Seja $n$ o mínimo dos graus dos polinômios em $J$ e $I \subseteq$ $R$ o ideal dos coeficientes lideres de polinômios de grau $n$ em $J$. Dado $t \in I$ existe um polinômio $g(X) \in J$ tal que $g(X)=t X^{n}+\cdots$, então $d(g(X))=\left(d(t)+f_{1} n t\right) X^{n}+\cdots$, como $d(J) \subseteq J$, então $\left(d(t)+f_{1} n t\right) \in I, \operatorname{logo} I$ é um $d$-ideal. Mas $R$ é $d$-simples e $I \neq 0, \operatorname{logo} I=R$. Portanto $J$ contém um polinômio mônico $g(X)$ de grau $n$, digamos $g(X)=X^{n}+g_{n-1} X^{n-1}+\cdots+g_{1} X+g_{0}$. Então

$$
\begin{gathered}
d(g(X))=\left(d\left(g_{n-1}\right) x^{n-1}+\cdots+d\left(g_{0}\right)\right)+\left(f_{0}+f_{1} \cdot X\right)\left(n X^{n-1}+g_{n-1}(n-1) X^{n-2}+\cdots+g_{1}\right)= \\
n f_{1} X^{n}+\left(d\left(g_{n-1}\right)+f_{0} n+(n-1) f_{1} g_{n-1}\right) X^{n-1}+\cdots
\end{gathered}
$$

Temos que $d(g(X))$ e $n f_{1} g(X) \in J$ então

$$
d(g(X))-n f_{1} g(X)=\left(d\left(g_{n-1}\right)+f_{0} n+(n-1) f_{1} g_{n-1}-n f_{1} g_{n-1}\right) X^{n-1}+\cdots \in J,
$$

que tem grau menor do que $n$. Logo $d(g(X))-n f_{1} g(X)=0$. Em particular $d\left(g_{n-1}\right)+f_{0} n-$ $f_{1} g_{n-1}=0 \mathrm{e}$

$$
d\left(-\frac{1}{n} g_{n-1}\right)=f_{1}\left(-\frac{1}{n} g_{n-1}\right)+f_{0},
$$

ou seja, $d(r)=f(r)$ para $r=-\frac{1}{n} g_{n-1}$, uma contradição.

Portanto $R[X]$ é $d$-simples.

Corolário 46. Seja $R d$-simples e suponha que $d$ não é sobrejetora em $R$, isto é, existe $r \in R$ tal que $d(y) \neq r$ para todo $y \in R$. Vamos estender $d$ em $R[X]$ definindo $d(X)=r$. Então $R[X]$ é $d$-simples.

Teorema 47. Seja $R$ um domínio e $X$ uma indeterminada. Suponha que $R$ é $d$-simples para alguma $d \in \operatorname{Der}(R)$. Tome $r \in R$ não nulo e estenda $d$ a uma derivação de $R[X]$ definindo $d(X)=r X$. Suponha que para todo inteiro não nulo $m$, a única solução em $R$ para a equação $d(y)=m y r$ seja $y=0$. Então:

1. $J$ é um $d$-ideal de $R[X]$ se e somente se $J=X^{n} R[X]$ para algum inteiro $n \geq 1$. 
2. $R\left[X, X^{-1}\right]$ é $d$-simples.

Demonstração: (1) Seja $J$ um $d$-ideal de $R[X]$, então, assim como na demonstração do teorema $45, J$ é um ideal principal gerado por um polinômio mônico $g(X)$. Considere $g(X)=X^{n}+$ $y_{n-1} X^{n-1}+\cdots+y_{1} X+a_{0}$ com $y_{i} \in R$ e $d(g)=n r X^{n}+\left(d\left(y_{n-1}\right)+(n-1) r y_{n-1}\right) X^{n-1}+\cdots$. Temos que $d(g)-n r g=0$ implica $d\left(y_{m}\right)=(n-m) r y_{m}$ para cada $m=0,1, \cdots, n-1$, o que por hipótese implica $y_{m}=0$. Portanto $g(X)=X^{n}$ e $J=X^{n} R[X]$.

A reciproca é clara.

(2)Pelo item 1, todo $d$-ideal primo de $R[X]$ intersecta o conjunto multiplicativo $U=\left\{X^{n}, n \geq 1\right\}$. Como $R\left[X, X^{-1}\right]=U^{-1} R[X]$ o resultado é evidente. 

CAPÍTULO

\section{4}

D-SIMPLICIDADE DE ÁLGEBRAS AFINS

\section{1 d-simplicidade em anéis de polinômios}

Teorema 48. Seja $K$ um corpo e $R=K\left[X_{1}, \ldots X_{n}\right]$ o anel de polinômios sobre $K$ em $n$ indeterminadas. Defina uma derivação $d \in \operatorname{Der}(R)$ da seguinte forma

$$
d\left(X_{1}\right)=1 \text { e } d\left(X_{i}\right)=X_{i} X_{i-1}+1 \text { para todo } \mathrm{i}=2,3, \ldots, \mathrm{n} .
$$

Então $R$ é $d$-simples.

Demonstração: Vamos fazer essa demonstração por indução. $\mathrm{O}$ caso $n=1$ é claro.

Suponha agora que $R^{\prime}=K\left[X_{1}, \ldots, X_{n-1}\right]$ é $d$-simples. Vamos estender nossa derivação tomando $d\left(X_{n}\right)=f\left(X_{n}\right)=X_{n} X_{n-1}+1 \in R^{\prime}\left[X_{n}\right]$. Considere $r \in R^{\prime}$ de grau $m$ em $X_{n-1}$, temos que

$$
d(r)=\frac{\partial r}{\partial X_{1}}+\sum_{i=2}^{n-1}\left(1+X_{i} X_{i-1}\right) \frac{\partial r}{\partial X_{i}}
$$

tem grau no máximo $m$ em $X_{n-1}$, entretanto $f(r)=r X_{n-1}+1$ tem grau $m+1$ em $X_{n-1}$. Consequentemente $d(r) \neq f(r)$ e pelo teorema $45, R=R^{\prime}\left[X_{n}\right]$ é $d$-simples.

Corolário 49. Seja $K$ um corpo e $T=K\left[X_{1}, \ldots\right]$ o anel de polinômios sobre $K$ em infinitas indeterminadas. Defina recursivamente uma derivação $d \in \operatorname{Der}(T)$ da seguinte forma

$$
d\left(X_{1}\right)=1 \text { e } \quad d\left(X_{i}\right)=X_{i} X_{i-1}+1 \text { para todo } \mathrm{i}=2,3, \ldots
$$

Então $T$ é $d$-simples.

Demonstração: Para cada $n \in \mathbb{N}$ seja $R_{n}=K\left[X_{1}, \ldots, X_{n}\right]$. Temos que $d\left(R_{n}\right) \subseteq R_{n}$ e $R_{n}$ é $d$-simples pelo resultado anterior. Seja $I$ um ideal de $T$. Teremos que $I \cap R_{n} \neq \emptyset$ para algum $n<\infty$ e se $d(I) \subseteq I$ então $d\left(I \cap R_{n}\right) \subseteq I \cap R_{n}$, o que nos daria uma contradição.

Seja $K$ um corpo e $t_{1}, \ldots, t_{n}$ indeterminadas. $\mathrm{O}$ anel $T=K\left[t_{1}, t_{1}^{-1}, \ldots, t_{n}, t_{n}^{-1}\right]$ é chamado o anel de polinômios de Laurent. Seja $T_{0}=K$ e para cada $n>0$ tome $T_{n}=K\left[t_{1}, t_{1}^{-1}, \ldots, t_{n}, t_{n}^{-1}\right]$. 
Lema 50. Seja $u$ uma unidade em $T_{n}(n \geq 1)$, então $u=a t_{1}^{\alpha_{1}} \cdots t_{n}^{\alpha_{n}}, \operatorname{com} a \in K$ e $\alpha_{i} \in \mathbb{Z}$.

Demonstração: Seja $R_{n}=K\left[t_{1}, \ldots, t_{n}\right]$ então qualquer elemento de $T_{n}$ pode ser escrito na forma $\left(t_{1}^{-\beta_{1}} \cdots t_{n}^{-\beta_{n}}\right) f$ com $f \in R_{n}$ e $\beta_{i} \geq 0$. Vamos escrever $u=\left(t_{1}^{-\gamma_{1}} \cdots t_{n}^{-\gamma_{n}}\right) g$ e seu inverso $u^{-1}=\left(t_{1}^{-\delta_{1}} \cdots t_{n}^{-\delta_{n}}\right) h$ com $g, h \in R_{n}$. Do produto $u u^{-1}=1$ obtemos a relação polinomial

$$
g h=t_{1}^{\gamma_{1}+\delta_{1}} \cdots t_{n}^{\gamma_{n}+\delta_{n}}
$$

essa equação só é satisfeita se $g$ pode ser expresso na forma $a t_{1}^{\alpha_{1}} \cdots t_{n}^{\alpha_{n}} \operatorname{com} a \in K$ e $\alpha_{i} \geq 0$. Portanto $u=a t_{1}^{\alpha_{1}-\beta_{1}} \cdots t_{n}^{\alpha_{n}-\beta_{n}}$, como desejado.

Teorema 51. Seja $K$ um corpo com $\operatorname{dim}_{\mathbb{Q}} K \geq n$ e sejam $\lambda_{1}, \cdots, \lambda_{n}$ elementos de $K$ não nulos e linearmente independentes sobre $\mathbb{Q}$. Seja $T_{0}=K$ e $\delta_{0}=0$ e para cada $m=1, \cdots, n$ considere $T_{m}=K\left[t_{1}, t_{1}^{-1}, \cdots, t_{m}, t_{m}^{-1}\right]$ e $\delta_{m}=\sum_{j=1}^{m} \lambda_{j} t_{j} \frac{\partial}{\partial t_{j}}$. Então $\delta_{m} \in \operatorname{Der}_{K}\left(T_{m}\right)$ e $T_{m}$ é $\delta_{m}$-simples para todo $m=0,1, \cdots, n$.

Demonstração: Vamos proceder por indução em $m$. O caso $m=0$ é claro. Suponha agora que $T_{m}$ é $\delta_{m}$-simples para todo $0 \leq m \leq n-1$. Considere $U=\left\{t_{m+1}^{l}, l \geq 0\right\} \subseteq T_{m}\left[t_{m+1}\right]$. Note que $U$ é multiplicativamente fechado e $T_{m+1}=U^{-1} T_{m}\left[t_{m+1}\right]$. Observe que $\delta_{m+1} \in \operatorname{Der}\left(T_{m}\left[t_{m+1}\right]\right)$ e para aplicar o teorema 47 basta mostrarmos que a única solução $y \in T_{m}$ para uma equação da forma $\delta_{m+1}(y)=s y \lambda_{m+1}$ é $y=0$, onde $s$ é um inteiro não nulo.

Suponha que $y \in T_{m}$ satisfaz $\delta_{m+1}(y)=s y \lambda_{m+1} \operatorname{com} s \neq 0$ inteiro. Temos que $\delta_{m+1}$ se restringe a $\delta_{m}$ em $T_{m}$ e $T_{m}$ é $\delta_{m}$-simples por hipótese de indução. Isto implica que $y=0$ ou $y$ é uma unidade em $T_{m}$, caso contrário $\langle y\rangle$ seria um $\delta_{m}$-ideal próprio.

Caso $y$ seja diferente de 0 , o lema anterior nos diz que $y=a t_{1}^{\alpha_{1}} \cdots t_{m}^{\alpha_{m}}$, para $\alpha_{i} \in \mathbb{Z}$ e $a \in K$ não nulo.

Então $\delta_{m}(y)=\sum_{i=1}^{m} \alpha_{j} \lambda_{j} y=s \lambda_{m+1} y$. Como $y \neq 0$ obtemos $\alpha_{1} \lambda_{1}+\alpha_{2} \lambda_{+} \cdots+\alpha_{m} \lambda_{m}-s \lambda_{m+1}=0$. Portanto $\alpha_{1}=\cdots=\alpha_{m}=s=0$ pois os $\lambda_{i}$ são linearmente independentes sobre $\mathbb{Q}$. O que contradiz $s \neq 0$. Concluímos então que $y=0$ é a unica solução e o resultado segue do teorema 47.

\subsection{Exemplos geométricos}

\subsubsection{O círculo e o cilindro}

Sejam $R=K\left[X_{1}, X_{2}\right] /\left(X_{1}^{2}+X_{2}^{2}-1\right)$ e $x_{i}$ a imagem de $X_{i}$ em $R$, ou seja $R=K\left[x_{1}, x_{2}\right]$ sujeito a relação $x_{1}^{2}+x_{2}^{2}=1$. Observe que $R$ é o anel de coordenadas de um círculo sobre o corpo $K$, um domínio regular de dimensão 1 e os seus ideais primos não nulos são todos maximais. Seja $d=x_{2} \frac{\partial}{\partial x_{1}}-x_{1} \frac{\partial}{\partial x_{2}}$. Note que $d \in \operatorname{Der}_{k}(R)$, pois $D=X_{2} \frac{\partial}{\partial X_{1}}-X_{1} \frac{\partial}{\partial X_{2}}$ é uma derivação em $K\left[X_{1}, X_{2}\right]$ que fixa o ideal $\left(X_{1}^{2}+X_{2}^{2}-1\right)$, já que $D\left(X_{1}^{2}+X_{2}^{2}-1\right)=0$. Portando $D$ dá origem à derivação $d$ no anel quociente $R$. Além disso $d\left(x_{1}\right) R+d\left(x_{2}\right) R=x_{2} R+x_{1} R=R$, então, pelo corolário $41, R$ não possui $d$-ideais maximais e portanto $R$ é $d$-simples. 
O anel $S=K\left[X_{1}, X_{2}, X_{3}\right] /\left(X_{1}^{2}+X_{2}^{2}-1\right)=K\left[x_{1}, x_{2}, X_{3}\right]=R\left[X_{3}\right]$ é o anel de coordenadas de um cilindro definido sobre o corpo $K$. Nosso objetivo é estender nossa derivação $d$ anterior para $S$ de forma que $S$ seja $d$-simples. Pelo corolário 46 basta provar que $d$ não é sobrejetora. Mais que isso, vamos provar que $d(F) \neq 1$ para todo $F \in R$.

De fato, seja $F \in R$ e suponha que $d(F)=1$. Temos que $F$ pode ser representado, de maneira única, como $F=f x_{2}+g \operatorname{com} f, g \in K\left[x_{1}\right]$. Para checar a existência dessa representação basta aplicar o algoritmo da divisão num representante de $F, H \in K\left[X_{1}, X_{2}\right]$ para obter $H=q\left(X_{1}\right) . X_{2}+$ $r\left(X_{1}\right)$ e tomar seu quociente por $X_{1}^{2}+X_{2}^{2}-1$. Além disso a unicidade segue pois se $F=a x_{2}+b$ é outra representação para $F$ teremos $(f-a) x_{2}+(g-b)=0$ em $R$ ou equivalentemente $(f-a)\left(X_{1}\right) X_{2}+(g-b)\left(X_{1}\right)=h\left(X_{1}, X_{2}\right)\left(X_{1}^{2}+X_{2}^{2}-1\right)$ em $K\left[X_{1}, X_{2}\right]$ para algum $h\left(X_{1}, X_{2}\right) \in K\left[X_{1}, X_{2}\right]$. Comparando o grau de $X_{2}$ na última equação, concluímos que $f-a=$ $0=g-b$.

Sendo assim, se $d(F)=d\left(f x_{2}+g\right)=1$, temos que

$$
\begin{gathered}
x_{2}\left(\frac{\partial f}{\partial x_{1}} x_{2}+\frac{\partial g}{\partial x_{1}}\right)-x_{1} f=1 \mathrm{e} \\
\frac{\partial g}{\partial x_{1}} x_{2}+\left(1-x_{1}^{2}\right) \frac{\partial f}{\partial x_{1}}-x_{1} f=1
\end{gathered}
$$

Pela unicidade de representação de 1 em $K\left[x_{1}, x_{2}\right]$, assim como fizemos com $F$, concluímos que $\frac{\partial g}{\partial x_{1}}=0$ e $\left(1-x_{1}^{2}\right) \frac{\partial f}{\partial x_{1}}-x_{1} f=1$. Consequentemente

$$
\left(1-X_{1}^{2}\right) \frac{\partial f\left(X_{1}\right)}{\partial X_{1}}-X_{1} f\left(X_{1}\right)=1
$$

Suponha que $f\left(X_{1}\right)=a X_{1}^{n}+\ldots$. Comparando os coeficientes líderes na equação acima teremos que $-(n+1) a=0$ e portanto $a=0$ pois estamos em característica 0 .

Portanto $f\left(X_{1}\right)=0$, absurdo. Com isso provamos:

Teorema 52. Seja $R=K\left[x_{1}, x_{2}\right]$, sujeito a relação $x_{1}^{2}+x_{2}^{2}=1$ e seja $S=R\left[X_{3}\right]$, onde $X_{3}$ é uma indeterminada livre. Considere a $K$-derivação em $S$ definida por

$$
d=x_{2} \frac{\partial}{\partial x_{1}}-x_{1} \frac{\partial}{\partial x_{2}}+\frac{\partial}{\partial X_{3}}
$$

Então $S$ é $d$-simples.

Pelo teorema acima temos que $d$ origina um campo vetorial tangente ao cilindro $C$ definido por $S$ que não se restringe a nenhuma subvariedade algébrica de $S$, isto é, $d$ não induz um campo vetorial tangente em nenhuma curva algébrica definida na superfície de $C$. Vamos explorar isso melhor no seguinte exemplo:

Considere $K=\mathbb{R}$. Seja $H$ a hélice contida no cilindro $C$ com os pontos dados parametricamente por $(\operatorname{sen}(t), \cos (t), t), t \in \mathbb{R}$. O vetor $\frac{\partial}{\partial t}(\operatorname{sen}(t), \cos (t), t)=(\cos (t),-\operatorname{sen}(t), 1)$ está na direção tangente a $H$ no ponto correspondente ao parâmetro $t$. Além disso $d$ induz em cada ponto $\left(x_{1}, x_{2}, x_{3}\right) \in C$ o vetor tangente $\left(x_{2},-x_{1}, 1\right)$ e portanto a cada ponto em $H$ correspondente a 
um parâmetro $t$ o vetor $(\cos (t),-\operatorname{sen}(t), 1)$ que é tangente a $H$. Concluímos então que $d$ induz um campo vetorial tangente à hélice e como $S$ é $d$-simples, $H$ não pode ser uma subvariedade algébrica de $C$.

Lema 53. Seja $S=K\left[X_{1}, X_{2}, X_{3}\right] /\left(X_{1}^{2}+X_{2}^{2}-1\right)$. Então $\Omega_{K}(S) \simeq S^{2}$ e existem derivações $d_{1}, d_{2}$

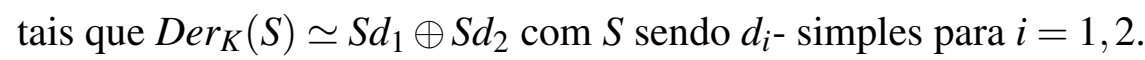

Demonstração: Considere $S=K\left[x_{1}, x_{2}, X_{3}\right]$, sujeito a $x_{1}^{2}+x_{2}^{2}=1$. Sabemos que $\Omega_{K}(S)$ é gerado por $d\left(x_{1}\right), d\left(x_{2}\right)$ e $d\left(X_{3}\right) \operatorname{com} d=d^{S}$ sendo a $K$-derivação universal. Seja $\omega=x_{2} d\left(x_{1}\right)-$ $x_{1} d\left(x_{2}\right) \in \Omega_{K}(S)$. Vamos mostrar que $\Omega_{K}(S)=\omega S \oplus d\left(X_{3}\right) S \simeq S^{2}$.

Seja $\delta_{1}=x_{2} \frac{\partial}{\partial x_{1}}-x_{1} \frac{\partial}{\partial x_{2}} \in \operatorname{Der}_{K}(S)$ e $h_{1}$ o único homomorfismo de $\Omega_{K}(S)$ para $S$ tal que $\delta_{1}=h_{1} d$. Temos que $h_{1}(\omega)=x_{2} \delta_{1}\left(x_{1}\right)-x_{1} \delta_{1}\left(x_{2}\right)=x_{2}^{2}+x_{1}^{2}=1$. Portanto $h_{1}$ é sobrejetor e $\Omega_{K}(S) \simeq$ $\omega S \oplus \operatorname{Ker}\left(h_{1}\right)$.

Tome agora $v=s_{1} d\left(x_{1}\right)+s_{2} d\left(x_{2}\right)+s_{3} d\left(X_{3}\right) \in \operatorname{Ker}\left(h_{1}\right), s_{i} \in S$. Temos que $h_{1}(v)=0=s_{1} \delta_{1}\left(x_{1}\right)+$ $s_{2} \delta_{1}\left(x_{2}\right)=s_{1} x_{2}-s_{2} x_{1}$, portanto $s_{1} x_{2}=s_{2} x_{1}$ o que implica $s_{1}=t x_{1}$ e $s_{2}=t x_{2}$ para algum $t \in S$. $\operatorname{Logo} v=t\left(x_{1} d\left(x_{1}\right)+x_{2} d\left(x_{2}\right)\right)+s_{3} d\left(X_{3}\right)$, além disso $x_{1} d\left(x_{1}\right)+x_{2} d\left(x_{2}\right)=\frac{1}{2} d\left(x_{1}^{2}+x_{2}^{2}\right)=\frac{d(1)}{2}=0$ de onde concluímos que $v=s_{3} d\left(X_{3}\right)$. Portanto $\Omega_{K}(S)=\omega S \oplus d\left(X_{3}\right) S$.

Seja agora $\delta_{2}=\frac{\partial}{\partial X_{3}}$, vamos provar que $\delta_{1}$ e $\delta_{2}$ geram $\operatorname{Der}_{K}(S)$. Temos que $\delta_{2}=h_{2} d$, onde $h_{2} \in \operatorname{Hom}_{S}\left(\Omega_{K}(S), S\right)$, seja $s \in S$ então $d(s)=r \omega+t d\left(X_{3}\right)$ para certos $r, t \in S$. Aplicando $h_{1}$ e $h_{2}$ em $d(s)$ obtemos, respectivamente, $\delta_{1}(s)=r$ e $\delta_{2}(s)=t$. Portanto $d(s)=\delta_{1}(s) \omega+\delta_{2}(s) d\left(X_{3}\right)$. Tomando então $\delta=h d \in \operatorname{Der}_{K}(S)$ qualquer, temos que $\delta(s)=h(\omega) \delta_{1}(s)+h\left(d\left(X_{3}\right)\right) \delta_{2}(s)$ para todo $s \in S$. Portanto $\delta=h(\omega) \delta_{1}+h\left(d\left(X_{3}\right)\right) \delta_{2}$ e $\delta_{1}$ e $\delta_{2}$ geram $\operatorname{Der}_{K}(S)$.

Mas $\operatorname{Der}_{K}(S) \simeq S^{2}$ pois $\Omega_{K}(S) \simeq S^{2}$, o que nos permite concluir que $\delta_{1}$ e $\delta_{2}$ formam uma base de $\operatorname{Der}_{K}(S)$.

Por fim tome $d_{1}=\delta_{1}+\delta_{2}$ e $d_{2}=\delta_{1}-\delta_{2}$. Pelo teorema anterior é fácil ver que $S$ é $d_{i}$-simples para $i=1,2$ e além disso, $\left\{d_{1}, d_{2}\right\}$ formam uma base para $\operatorname{Der}_{K}(S)$.

\subsubsection{O toro real}

Seja $R=\mathbb{R}\left[X_{1}, X_{2}, Y_{1}, Y_{2}\right] /\left(X_{1}^{2}+Y_{1}^{2}-1, X_{2}^{2}+Y_{2}^{2}-1\right)=\mathbb{R}\left[x_{1}, x_{2}, y_{1}, y_{2}\right]$ o anel de coordenadas do toro real $T^{2}$ visto como uma superfície bidimensional em quatro dimensões.

Dados $a, b \in(R)$ a aplicação

$$
d=a y_{1} \frac{\partial}{\partial x_{1}}-a x_{1} \frac{\partial}{\partial y_{1}}+b y_{2} \frac{\partial}{\partial x_{2}}-b x_{2} \frac{\partial}{\partial y_{2}},
$$

é uma $\mathbb{R}$-derivação de $R$ em $R$, pois a derivação $D=a Y_{1} \frac{\partial}{\partial X_{1}}-a X_{1} \frac{\partial}{\partial Y_{1}}+b Y_{2} \frac{\partial}{\partial X_{2}}-b X_{2} \frac{\partial}{\partial Y_{2}}$ em $K\left[X_{1}, X_{2}, Y_{1}, Y_{2}\right]$ é tal que $D\left(X_{1}^{2}+Y_{1}^{2}-1\right)=D\left(X_{2}^{2}+Y_{2}^{2}-1\right)=0$ e portanto $D$ fixa o ideal $\left\langle X_{1}^{2}+Y_{1}^{2}-1, X_{2}^{2}+Y_{2}^{2}-1\right\rangle$ e dá origem à derivação $d$ no quociente $R$. Vamos supor que $a b \neq 0$. Temos então:

Teorema 54. R é $d$-simples se e somente se $\frac{a}{b}$ é irracional. 
Demonstração: Pelo teorema 39, podemos estender $d$ a uma derivação, também denotada por $d$ em $S=\mathbb{C}\left[x_{1}, x_{2}, y_{1}, y_{2}\right]$ e $S$ é $d$-simples se e somente se $R$ o é.

Seja $T=\mathbb{C}\left[t_{1}, t_{1}^{-1}, t_{2}, t_{2}^{-1}\right], T$ é isomorfo a $S$ via o isomorfismo $\sigma$ que age da seguinte forma:

$$
\begin{aligned}
& x_{1}+i y_{1} \mapsto t_{1}, \quad x_{1}-i y_{1} \mapsto t_{1}^{-1}, \\
& x_{2}+i y_{2} \mapsto t_{2}, \quad x_{2}-i y_{2} \mapsto t_{2}^{-2} .
\end{aligned}
$$

Ou equivalentemente:

$$
\begin{array}{ll}
x_{1} \mapsto \frac{t_{1}+t_{1}^{-1}}{2} & y_{1} \mapsto \frac{t_{1}-t_{1}^{-1}}{2 i}, \\
x_{2} \mapsto \frac{t_{2}+t_{2}^{-1}}{2} & y_{2} \mapsto \frac{t_{2}-t_{2}^{-1}}{2 i} .
\end{array}
$$

Suponha que esse isomorfismo leve $d$ em $\delta \in \operatorname{Der}(T)$ onde $\delta=\sigma^{-1} \circ d \circ \sigma$. Temos que $d\left(x_{1}+i y_{1}\right) \mapsto \delta\left(t_{1}\right)$, mas $d\left(x_{1}+i y_{1}\right)=-i a\left(x_{1}+i y_{1}\right)$. Portanto $\delta\left(t_{1}\right)=-i a t_{1}$ e similarmente $\delta\left(t_{2}\right)=-i b t_{2}$.

Quando $\frac{a}{b}$ é irracional temos que $-i a$ e $-i b$ são linearmente independentes sobre $\mathbb{Q}$ e pelo teorema 51, $T$ é $\delta$-simples. Consequentemente $S$ e $R$ são $d$-simples.

Já no caso em que $\frac{a}{b}$ é racional, podemos escrever $\frac{a}{b}=\frac{m}{n} \operatorname{com} m$ e $n$ inteiros não nulos. Note que

$$
\delta\left(t_{1}^{n}-t_{2}^{m}\right)=-i\left(n a t_{1}^{n}-m b t_{2}^{m}\right)=-i n a\left(t_{1}^{n}-t_{2}^{m}\right) .
$$

Ou seja $t_{1}^{n}-t_{2}^{m}$ é um $\delta$-ideal de $T$. Consequentemente o ideal correspondente em $S$ e sua contração em $R$ serão $d$-ideais de $S$ e $R$ respectivamente.

Vamos ver explicitamente um $d$-ideal de $R$. Para cada $n \in \mathbb{Z}$ denote por $T_{n}(X)$ a função de Tchebychev $\cos \left(n \cos ^{-1}(X)\right)$. Temos que $T_{n}(X) \in \mathbb{Z}[X]$. Dado $n$ inteiro, defina

$$
S_{n}(X)=\frac{\operatorname{sen}\left(n\left(\cos ^{-1}(X)\right)\right)}{\operatorname{sen}\left(\cos ^{-1}(X)\right)}
$$

Note que $\frac{\partial T_{n}(X)}{\partial X}=n S_{n}(X)$, portanto $S_{n}(X)$ é um polinômio e

$$
\frac{\partial S_{n}(X)}{\partial X}=\frac{X S_{n}(X)-n T_{n}(X)}{1-X^{2}} \text { também o é. }
$$

Suponha que $\frac{a}{b}=\frac{m}{n}$ para $m$ e $n$ inteiros não nulos. Sejam $F\left(x_{1}, x_{2}\right)=T_{n}\left(x_{1}\right)-T_{m}\left(x_{2}\right)$ e $G\left(x_{1}, x_{2}, y_{1}, y_{2}\right)=y_{1} S_{n}\left(x_{1}\right)-y_{2} S_{m}\left(x_{2}\right)$. Aplicando $d$ em $F$ e $G$ obtemos

$$
d(F)=n a y_{1} S_{n}\left(x_{1}\right)-m b y_{2} S_{m}\left(x_{2}\right)=n a G
$$

$\mathrm{e}$

$$
\begin{aligned}
& d(G)=-a x_{1} S_{n}\left(x_{1}\right)+\frac{a y_{1}^{2}\left(x_{1} S_{n}\left(x_{1}\right)-n T_{n}\left(x_{1}\right)\right)}{1-x_{1}^{2}}+b x_{2} S_{m}\left(x_{2}\right)-\frac{b y_{2}^{2}\left(x_{2} S_{m}\left(x_{2}\right)-m T_{m}\left(x_{2}\right)\right)}{1-x_{2}^{2}}= \\
= & a\left(\frac{y_{1}^{2}}{1-x_{1}^{2}}-1\right)\left(x_{1} S_{n}\left(x_{1}\right)-n T_{n}\left(x_{1}\right)\right)-n a T_{n}\left(x_{1}\right)-b\left(\frac{y_{2}^{2}}{1-x_{2}^{2}}-1\right)\left(x_{2} S_{m}\left(x_{2}\right)-m T_{m}\left(x_{2}\right)\right)+m b T_{m}\left(x_{2}\right)=
\end{aligned}
$$




$$
a\left(x_{1}^{2}+y_{1}^{2}-1\right) \frac{\partial S_{n}\left(x_{1}\right)}{\partial x_{1}}-b\left(x_{2}^{2}+y_{2}^{2}-1\right) \frac{\partial S_{m}\left(x_{2}\right)}{\partial x_{2}}-n a F=-n a F
$$

Portanto o ideal em $R$ gerado por $F$ e $G$ é um $d$-ideal.

Geometricamente esse $d$-ideal corresponde à subvariedade de $T^{2}$ cujo pontos estão sobre duas famílias de curvas dadas parametricamente por

$$
\left(\cos (t), \operatorname{sen}(t), \cos \left(\frac{2 N \pi+n t}{m}\right), \operatorname{sen}\left(\frac{2 N \pi+n t}{m}\right)\right)
$$

$\mathrm{e}$

$$
\left(\cos (t),-\operatorname{sen}(t), \cos \left(\frac{2 N \pi+n t}{m}\right),-\operatorname{sen}\left(\frac{2 N \pi+n t}{m}\right)\right)
$$

$\operatorname{com} t \in \mathbb{R}$ e $N \in \mathbb{Z}$.

Essas curvas são espirais ao longo da superfície $T^{2}$ e são periódicas.

Além disso $d$ induz um campo vetorial tangente na curva $W \subseteq T^{2}$ cujo pontos são descritos parametricamente por

$$
\left(\cos (t), \operatorname{sen}(t), \cos \left(\frac{b t}{a}\right), \operatorname{sen}\left(\frac{b t}{a}\right)\right) .
$$

Note que essa curva é periódica se, e somente se, $\frac{a}{b}$ é racional.

Caso $\frac{a}{b}$ seja irracional, suponha que $W$ é uma subvariedade algébrica de $T^{2}$. Então $d$ induz um campo vetorial em $W$ que é tangente em cada ponto de $W$. Isso implica que o ideal correspondente a $W$ é um $d$-ideal de $R$. Mas $R$ é $d$-simples, logo $W$ não é um conjunto algébrico. Isso pode ser provado analiticamente usando o fato de que pontos de $W$ são densos na superfície do toro quando $\frac{a}{b}$ é irracional.

Lema 55. Mantendo a notação do teorema anterior, temos que $\Omega_{\mathbb{R}}(R) \simeq R^{2}$ e $\operatorname{Der}_{\mathbb{R}}(R)$ admite uma base livre $\left\{d_{1}, d_{2}\right\}$ tal que $R$ é $d_{i}$-simples para $i=1,2$.

Demonstração: Tome $d_{i}=y_{i} \frac{\partial}{\partial x_{i}}-x_{i} \frac{\partial}{\partial y_{i}} \operatorname{com} i=1,2$. Seja $d=d_{\mathbb{R}}^{R}$ a $\mathbb{R}$-derivação universal $R \rightarrow \Omega_{\mathbb{R}}(R)$. Então existem únicos homomorfismos $h_{i} \in \operatorname{Hom}\left(\Omega_{\mathbb{R}}(R), R\right)$ tais que $d_{i}=h_{i} \circ d$.

Seja $w_{i}=y_{i} d\left(x_{i}\right)-x_{i} d\left(y_{i}\right)$, então $h_{1}\left(w_{1}\right)=h_{2}\left(w_{2}\right)=1$, logo $h_{1}$ e $h_{2}$ são sobrejetores e

$$
\Omega_{\mathbb{R}}(R) \simeq w_{1} R \oplus \operatorname{Ker}\left(h_{1}\right) .
$$

Vamos mostrar que $\operatorname{Ker}\left(h_{1}\right)=w_{2} R$. Certamente $w_{2} R \subseteq \operatorname{Ker}\left(h_{1}\right)$, pois $h_{1}\left(w_{2}\right)=0$.

Suponha agora que $v \in \operatorname{Ker}\left(h_{1}\right)$. Então $v=r_{1} d\left(x_{1}\right)+s_{1} d\left(y_{1}\right)+r_{2} d\left(x_{2}\right)+s_{2} d\left(y_{2}\right)$ para $r_{i}, s_{i} \in R$ e $h_{1}(v)=r_{1} d_{1}\left(x_{1}\right)+s_{1} d_{1}\left(y_{1}\right)=r_{1} y_{1}-s_{1} x_{1}=0, \log r_{1} y_{1}=s_{1} x_{1}$ e portanto $r_{1}=t x_{1}$ e $s_{1}=t y_{1}$, disso segue que $t\left(x_{1} d\left(x_{1}\right)+y_{1} d\left(y_{1}\right)\right)=0$. Consequentemente $v=r_{2} d\left(x_{2}\right)+s_{2} d\left(y_{2}\right)$ e $\operatorname{ker}\left(h_{1}\right)$ pode ser gerado por $d\left(x_{2}\right)$ e $d\left(y_{2}\right)$. Multiplicando por $d\left(x_{2}\right)$ e aplicando $d$ na relação $x_{2}^{2}+y_{2}^{2}=1$ obtemos, respectivamente, que $d\left(x_{2}\right)=x_{2}^{2} d\left(x_{2}\right)+y_{2}^{2} d\left(x_{2}\right)$ e $0=x_{2} d\left(x_{2}\right)+y_{2} d\left(y_{2}\right)$. Dessas duas relações segue que

$$
d\left(x_{2}\right)=y_{2}\left(y_{2} d\left(x_{2}\right)-x_{2} d\left(y_{2}\right)\right)=y_{2} w_{2} \quad \text { e } \quad d\left(y_{2}\right)=-x_{2} w_{2} .
$$

Portanto $\operatorname{Ker}\left(h_{1}\right)=R w_{2}$ e $\Omega_{\mathbb{R}}(R) \simeq R w_{1} \oplus R w_{2} \simeq R^{2}$.

Por fim é claro que $\left\{d_{1}, d_{2}\right\}$ é uma base para $\operatorname{Der}(R)$. Tome $a, b \in \mathbb{R}$ números irracionais distintos e defina $D_{1}=d_{1}+a d_{2}$ e $D_{2}=d_{1}+b d_{2}$. Pelo teorema $54, R$ é $D_{i}$-simples como $i=1,2$, além disso é evidente que $\left\{D_{1}, D_{2}\right\}$ é uma base para $\operatorname{Der}(R)$. 


\subsubsection{Hipersuperfícies}

Seja $K$ um corpo e $R=K\left[X_{1}, \cdots, X_{n}\right] /(f)=K\left[x_{1}, \cdots, x_{n}\right]$ o anel de coordenadas de uma hipersuperfície. Denote por $J$ a matriz jacobiana $\left(\frac{\partial f}{\partial X_{1}}, \cdots, \frac{\partial f}{\partial X_{n}}\right)_{1 \times n}$ e $J^{T}$ sua transposta.

Dada uma matriz antissimétrica $A_{n \times n}$ com entradas em $R$ seja $\left(a_{1}, \cdots, a_{n}\right)=J A$, então

$$
\sum_{j=1}^{n} a_{j} \frac{\overline{\partial f}}{\partial X_{j}}=J A J^{T}=0
$$

o que significa que a aplicação dada por $d=\sum_{j=1}^{n} a_{j} \frac{\partial}{\partial x_{j}}$ é uma $K$-derivação de $R$. Ou seja, toda matriz $n \times n$ antissimétrica dá origem a uma $K$-derivação de $R$, pois a derivação $D=\sum_{j=1}^{n} a_{j} \frac{\partial}{\partial X_{j}}$ em $K\left[X_{1}, \cdots, X_{n}\right]$ é tal que $D(f)=0$, o que dá origem à derivação $d$ no quociente $R$. Vamos mostrar que quando $R$ é regular temos uma correspondência entre essas matrizes e $\operatorname{Der}_{K}(R)$.

Lema 56. Seja $R=K\left[X_{1}, \cdots, X_{n}\right] /(f)=K\left[x_{1}, \cdots, x_{n}\right]$ e suponha que $R$ é um domínio regular. Seja $d \in \operatorname{Der}_{K}(R)$, então existe uma matriz antissimétrica $A$ tal que $\left(d\left(x_{1}\right), \cdots, d\left(x_{n}\right)\right)=J A$.

Demonstração: Temos duas equações em relação as entradas de $J$

$$
\sum_{i=1}^{n} d\left(x_{i}\right) \frac{\overline{\partial f}}{\partial X_{i}}=0, \text { pois } d \text { é uma derivação de } R
$$

$\mathrm{e}$

$$
\sum_{i=1}^{n} r_{i} \frac{\overline{\partial f}}{\partial X_{i}}=1 \text {, para alguns } r_{i} \in R, \text { pois } R \text { é regular. }
$$

A equação 4.1 vale pois $d$ dá origem a uma derivação $D$ em $K\left[X_{1}, \cdots, X_{n}\right]$ que fixa o ideal $(f)$ e pode ser escrita como $\sum_{i=1}^{n} D\left(X_{i}\right) \frac{\partial}{\partial X_{i}}$ e a equação 4.2 segue do corolário 16. Para cada $j=1, \cdots, n$, multiplicando $d\left(x_{j}\right)$ pela equação 4.2 obtemos

$$
\begin{gathered}
d\left(x_{j}\right)=\sum_{i=1}^{n} r_{i} d\left(x_{j}\right) \frac{\overline{\partial f}}{\partial X_{i}}=\sum_{i=1}^{n} r_{i} d\left(x_{j}\right) \frac{\overline{\partial f}}{\partial X_{i}}-\underbrace{\sum_{i=1}^{n} r_{j} d\left(x_{i}\right) \frac{\overline{\partial f}}{\partial X_{i}}}_{=0}, \text { pela equação } 4.1 \\
d\left(x_{j}\right)=\sum_{i=1}^{n}\left(r_{i} d\left(x_{j}\right)-r_{j} d\left(x_{i}\right)\right) \frac{\overline{\partial f}}{\partial X_{i}} .
\end{gathered}
$$

Agora para cada $j=1, \cdots, n-1$ e $i=j+1, \cdots, n$ defina $a_{i j}=r_{i} d\left(x_{j}\right)-r_{j} d\left(x_{i}\right)$. Então $\left(d\left(x_{1}\right), \cdots, d\left(x_{n}\right)\right)=A J$ onde $A=\left(\begin{array}{ccccc}0 & -a_{12} & -a_{13} & \cdots & -a_{1 n} \\ a_{12} & 0 & -a_{23} & \cdots & -a_{2 n} \\ a_{13} & a_{23} & 0 & \cdots & -a_{3 n} \\ \vdots & & & \vdots & \vdots \\ a_{1 n} & a_{2 n} & a_{3 n} & \cdots & 0\end{array}\right) . \mathbf{\square}$

No caso do círculo no lema 53 mostramos que $\Omega_{K}(R)$ é livre. Se $\Omega_{K}(R)$ é um módulo livre, o corolário 42 nos garante que $R$ possui uma derivação $d$ que não admite $d$-ideais maximais. No próximo teorema vamos melhorar esse resultado para o anel de coordenadas de certas hipersuperfícies. 
Teorema 57. Seja $R=K\left[X_{1}, X_{2}, X_{3}\right] /(f)=K\left[x_{1}, x_{2}, x_{3}\right]$ um domínio regular. Então $\Omega_{K}(R) \simeq R^{2}$ se e somente se $R$ admite uma derivação $d$ que não deixa $d$-ideais maximais invariantes.

Demonstração: Para $i=1,2,3$ denote $\frac{\partial \bar{f}}{\partial X_{i}}$ por $f_{i}$ e $\left(f_{1}, f_{2}, f_{3}\right)$ em $R^{3}$ por $y$.

Pela proposição 11 , temos que $\Omega_{K}(R) \simeq R^{3} / y R^{3}$, então basta mostrarmos que $y$ faz parte de uma base de $R^{3}$.

Seja $d \in \operatorname{Der}_{K}(R)$, pelo lema 56

$$
\left(d\left(x_{1}\right), d\left(x_{2}\right), d\left(x_{3}\right)\right)=\left(f_{1}, f_{2}, f_{3}\right)\left(\begin{array}{ccc}
0 & -a_{12} & -a_{13} \\
a_{12} & 0 & -a_{23} \\
a_{13} & a_{23} & 0
\end{array}\right)
$$

$\operatorname{com} a_{12}=r_{2} d\left(x_{1}\right)-r_{1} d\left(x_{2}\right), a_{13}=r_{3} d\left(x_{1}\right)-r_{1} d\left(x_{3}\right)$ e $a_{23}=r_{3} d\left(x_{2}\right)-r_{2} d\left(x_{3}\right)$ e $r_{1}, r_{2}, r_{3} \in R$ satisfazendo $r_{1} f_{1}+r_{2} f_{2}+r_{3} f_{3}=1$. Suponha agora que $d$ não deixa nenhum ideal maximal invariante. Pelo corolário 41 existem $s_{1}, s_{2}, s_{3} \in R$ tais que $s_{1} d\left(x_{1}\right)+s_{2} d\left(x_{2}\right)+s_{3} d\left(x_{3}\right)=1$.

Considere a matriz $A=\left(\begin{array}{ccc}s_{1} & s_{2} & s_{3} \\ f_{1} & f_{2} & f_{3} \\ a_{23} & -a_{13} & a_{12}\end{array}\right)$, então

$$
\begin{aligned}
\operatorname{det}(A) & =s_{1}\left(f_{2} a_{12}+f_{3} a_{13}\right)+s_{2}\left(f_{3} a_{12}+f_{2} a_{23}\right)+s_{3}\left(-f_{1} a_{13}-f_{2} a_{23}\right) \\
& =s_{1} d\left(x_{1}\right)+s_{2} d\left(x_{2}\right)+s_{3} d\left(x_{3}\right)=1 .
\end{aligned}
$$

Ou seja, $A$ é invertível em $R$ e portanto suas linhas formam uma base de $R^{3}$, de onde concluímos que $y$ faz parte de uma base de $R^{3}$ e $\Omega_{K}(R) \simeq R^{2}$.

A outra implicação segue diretamente do corolário 42 .

Lema 58. Seja $R=K\left[X_{1}, \cdots, X_{n}\right] /(f)=K\left[x_{1}, \cdots, x_{n}\right]$ um domínio regular e suponha que $n \geq 2$ é par. Então $R$ admite uma derivação que não deixa nenhum ideal maximal invariante.

Demonstração: Seja $n=2 m \operatorname{com} m \geq 1$. Para cada $j=1, \cdots, 2 m$ denote $\frac{\overline{\partial f}}{\partial X_{j}}$ por $f_{j}$. Como $R$ é regular, $\left(f_{1}, \cdots, f_{2 m}\right) R=R$. Considere a matriz $2 m \times 2 m$ antissimétrica $A$ cuja as entradas são dadas por $a_{(2 i-1)(2 i)}=1, a_{(2 i)(2 i-1)}=-1$ para $i=1, \cdots, m$ e 0 nas outras posições, ou seja

$$
A=\left(\begin{array}{ccccc}
0 & -1 & 0 & 0 & \cdots \\
1 & 0 & 0 & 0 & \ldots \\
0 & 0 & 0 & -1 & \\
0 & 0 & 1 & 0 & \\
\vdots & & & & \vdots
\end{array}\right)
$$

Então, pelo lema 56, $\left(d\left(x_{1}\right), \cdots, d\left(x_{2 m}\right)\right)=\left(f_{1}, \cdots, f_{2 m}\right) A=\left(f_{2},-f_{1}, f_{4},-f_{3}, \cdots, f_{2 m},-f_{2 m-1}\right)$ é uma derivação de $R$ e $\left(d\left(x_{1}\right), \cdots, d\left(x_{2 m}\right)\right) R=\left(f_{1}, f_{2}, \cdots, f_{2 m}\right) R=R$. Portanto $d$ não deixa ideais maximais invariantes, pelo corolário 41.

Geometricamente, o lema anterior nos diz que toda hipersuperfície não singular em um espaço afim de dimensão par admite um campo vetorial tangente que não se anula em nenhum ponto. 
Nem todo domínio regular $R$, finitamente gerado como $\mathbb{Q}$-álgebra, admite uma derivação $d$ tal que $R$ seja $d$-simples. Vamos ver isso no seguinte exemplo.

Exemplo 59. Para cada $n \geq 1$, seja $R_{n}=\mathbb{R}\left[X_{0}, X_{1}, \cdots, X_{n}\right] /\left(X_{0}^{2}+X_{1}^{2}+\cdots+X_{n}^{2}-1\right)$. Note que $R_{n}$ é um domínio regular $n$-dimensional, o anel de coordenadas da $n$-esfera real $S^{n}$.

Quando $n$ é par, digamos $n=2 m$ o "Teorema da bola cabeluda"(MILNOR, 1978) nos garante que todo campo vetorial suave, tangente à esfera $S^{2 m}$ tem um ponto singular. Portando toda derivação $d \in \operatorname{Der}\left(R_{n}\right)$ deixa pelo menos um ideal maximal invariante, consequentemente o corolário 42 nos garante que $\Omega_{\mathbb{R}}\left(R_{n}\right)$ não admite um somando direto de posto 1 .

Agora, quando $n$ é impar, o lema anterior nos diz que existe uma $\mathbb{R}$-derivação que não deixa ideais maximais invariantes e portanto $S^{n}$ admite um campo vetorial tangente que não se anula em qualquer ponto e nesse caso $\Omega_{\mathbb{R}}\left(R_{n}\right)$ admite um somando direto de posto 1 . 

ARCHER, J. Derivations on commutative rings and projective modules over skew polynomial rings. Tese (Doutorado) - University of Leeds, 1981. Citado nas páginas 11, 13 e 18.

ATIYAH, M. F.; MACDONALD, I. G. Introduction to Commutative Algebra. Great Britain: Addison-Wesley Publishing Company, 1969. Citado na página 32.

BORGES, H.; TENGAN, E. Álgebra comutativa em quatro movimentos. Rio de Janeiro: IMPA, 2015. Citado nas páginas 23 e 30.

BRUMATTI, P.; LEQUAIN, Y.; LEVCOVITZ, D. Differential simplicity in polynomial rings and algebraic independence of power series. Journal of the London Mathematical Society, v. 68 , p. 615-630, 2003. Citado na página 17.

COUTINHO, S. C. Nonholonomic simple $\mathscr{D}$-modules from simple derivations. Glasgow Mathematical Journal, v. 49, p. 11-21, 2007. Citado na página 17.

On the classification of simple quadratic derivations over the affine plane. Journal of Algebra, v. 319, p. 4249-4274, 2008. Citado na página 17.

On some foliations arising in $\mathscr{D}$-module theory. Geom Dedicata, v. 164, p. 27-45, 2013. Citado na página 17.

COUTINHO, S. C.; LEVCOVITZ, D. On the differential simplicity of affine rings. Proceedings of American Mathematical Society, v. 142, p. 34-37, 2014. Citado na página 17.

GOODEARL, K.; WARFIELD, J. R. B. An introduction to noncommutative Noetherian rings. second. [S.1.]: Cambridge University Press, 2004. v. 61. (London Mathematical Society Student Texts, v. 61). Citado na página 17.

HARTSHORNE, R. Algebraic Geometry. Nova Iorque: Springer-Verlag, 1977. Citado na página 24.

MILNOR, J. Analytic proofs of the "hairy ball theorem"and the brouwer fixed point theorem. The American Mathematical Monthly, v. 85, n. 7, p. 521-524, 1978. Citado na página 45.

OLIVEIRA, C. d. Sobre derivações simples e folheações holomorfas sem solução algébrica. Tese (Doutorado) — UFRJ/COPPE, 2012. Citado na página 17.

ZARISKI, O.; SAMUEL, P. Commutative Algebra. [S.1.]: D. VAN NOSTRAND COMPANY, INC, 1958. Citado na página 24. 


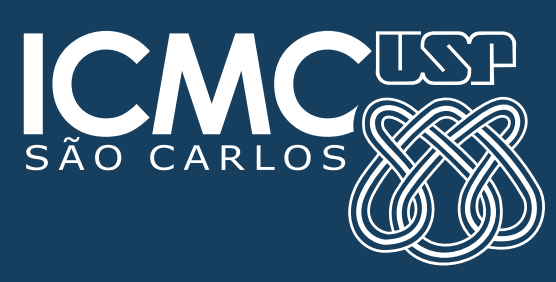

